\title{
A Method of Refinement in UML-B
}

\author{
Mar Yah Said • Michael Butler • Colin Snook
}

Received: date / Accepted: date

\begin{abstract}
UML-B is a 'UML-like' graphical front end for Event-B that provides support for object-oriented and state-machine modelling concepts, which are not available in Event-B. In particular, UML-B includes class diagram and state-machine diagram editors with automatic generation of corresponding Event-B. In Event-B, refinement is used to relate system models at different abstraction levels. The same refinement concepts are also applicable in UML-B but require special consideration due to the higher-level modelling concepts. In previous work we described a case study to introduce support for refinement in UML-B. We now provide a more complete presentation of the technique of refinement in UML-B including a formalisation of the refinement rules and a definition of the extensions to the abstract syntax of UML-B notation. The provision of gluing invariants to discharge the proof obligations associated with a refinement is a significant step in providing verifiable models. We discuss and compare two approaches for constructing gluing invariants in the context of UML-B refinement.
\end{abstract}

Keywords Visual modelling languages · Formal specification · UML-B · Event-B · Class Diagram · State Machine

Mar Yah Said

FSKTM, Universiti Putra Malaysia, Malaysia

Tel.: +006-03-84971772

Fax: +006-03-84966577

E-mail: maryah@upm.edu.my

Michael Butler

ECS, University of Southampton, UK

E-mail: mjb@ecs.soton.ac.uk

Colin Snook

ECS, University of Southampton, UK

E-mail: cfs@ecs.soton.ac.uk

\section{Introduction}

UML-B [1] is a graphical front end for Event-B [4] that has some similarity with UML $[2,3]$. Event-B is a state-based formalism with support for refinement and proof. UML-B supports class and state machine diagrams; concepts that are not supported in Event-B. UML-B was originally based on B [6] (now often referred to as 'Classical B') which is a method for verified software development based on the Abstract Machine Notation (AMN) and set theory. The UML-B tool, supporting the current, Event-B based, UML-B notation, is a plug-in to the Rodin platform $[5,10]$. Event-B models are generated from UML-B models by the UML$\mathrm{B}$ tool. The Rodin tools are used to report back any static verification errors and then generate and prove proof obligations associated with the generated EventB models. The purpose of UML-B is twofold. Firstly, it provides two essential modelling concepts that are absent in Event-B; object-orientation and event sequencing. Secondly, it provides a more approachable and efficient modelling notation especially for those familiar with UML.

Event-B was developed as an alternative to Classical B in order to support modelling at a system's level. Event-B distinguishes between contexts and machines so that machines can be re-used with different configurations. A context contains static configuration including given sets, constants and properties (including type) of the constants. A machine, which may see several contexts, contains state variables, invariant properties of the variables and events that update the variables. Each event is a guarded action which may fire when its guard is true and then executes actions which change the state of the variables. The guards of an event are predicates over the variables of the machine and any 
local parameters of the event. The actions are a set of parallel substitutions which change the state of the variables.

Refinement $[6,8]$ is the process of building a model gradually by making it more and more precise while verifying that each refined model satisfies the abstract behaviour. The advantage of starting with an abstract model is that important properties can be defined in a simple model which is therefore less likely to contain mistakes. Refinements then introduce detail in steps which are guaranteed to preserve the important properties. Refinement in Event-B involves refining the model state by adding or substituting variables and refining events into corresponding concrete versions, and by adding new events. The abstract state variables, $x$, and the concrete state variables, $y$, are linked together by a predicate called a gluing invariant $J(x, y)$. The gluing invariant provides the link between the abstract and concrete representations of state that is needed to verify that each abstract event is a correct simulation of its concrete version. Providing sufficient but provable gluing invariants can be a significant task. The refinement concepts of Event-B must be supported in UML-B for it to be a successful front-end to Event-B.

An alternative step to refinement is decomposition of a model into parts which is important for scalability. Mechanisms for decomposing Event-B models that retain composition of verification have been developed and work to support these in UML-B is currently underway. Decomposition is not covered in this paper.

Our previous work in [25] detailed a case study that was used to investigate support for class and state-machine refinement in UML-B. We now provide a more complete presentation of the technique of refinement in UML-B. The contributions of this paper are:

- a description of the intuition behind UML-B refinement,

- a formalisation of the UML-B refinement rules using the Event-B notation,

- a definition of the extensions to the abstract syntax (meta-model) of the UML-B notation that were needed to support refinement,

- a discussion on constructing gluing invariants in the context of UML-B refinement.

The organisation of the paper is as follows. In Section 2 we give some background on UML-B and the generated Event-B excluding refinement. Section 3 gives an intuitive overview of UML-B refinement in terms of data refinement and event refinement. Section 4 gives a formalisation of the syntactic rules for class diagram and state machine refinement. Section 5 describes the extensions to the abstract syntax (i.e. meta-model) of the UML-B notation. Section 6 provides an overview of the ATM case study development. Section 7 discusses two alternative approaches to constructing gluing invariants. Section 9 concludes the paper. Section 8 presents some related work.

\section{Background of UML-B and Generated Event-B}

There are four kinds of diagrams in UML-B. They are package, context, class and state machine diagrams. A package diagram shows the structure and relationships between components (machines and contexts) in a project. A context diagram is similar to a class diagram but contains constant data and structured types. A context diagram described a context. A machine is specified by a class diagram and state machine diagram(s) representing data structures that may be changed by events or transitions. Events may be attached to classes in a class diagram. Events can also be represented by the transitions in a state machine diagram. The semantics of a UML-B model are given by the Event-B generated by the UML-B tool according to a set of translation rules. The following subsections describe more about the package, context, class and state machine diagrams.

\subsection{Package Diagram}

A package diagram defines the relationships between UML-B machines and contexts in a project. Fig. 1 shows an example of a package diagram. The package diagram consists of machines $M 1$ and $M 2$, and refinement relationship between them. It also contains contexts $C X 1$ and $C X 2$, and the extension relationship between them and which contexts are seen by the machines.

\subsection{Context Diagram}

A context diagram defines the static part of a model. A context diagram may have classtypes. Each classtype may have attributes and associations. An attribute defines a constant that has a data value for each instance of a classtype. An association is a special case of an attribute that defines a relationship between two classtypes. Fig. 2 shows an example of a context diagram. The classtype CUSTOMER has an attribute, ident, and an association accounts, with the classtype $B A N K$. The $1 . .1$ target multiplicity of the association accounts indicates that it is a total function. Axioms and theorems ${ }^{1}$ may be attached to a classtype in which

1 A theorem is a predicate that must be proved from the preceding predicates. 


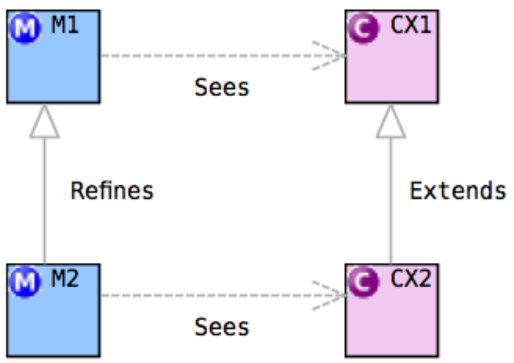

Fig. 1 Example of Package Diagram

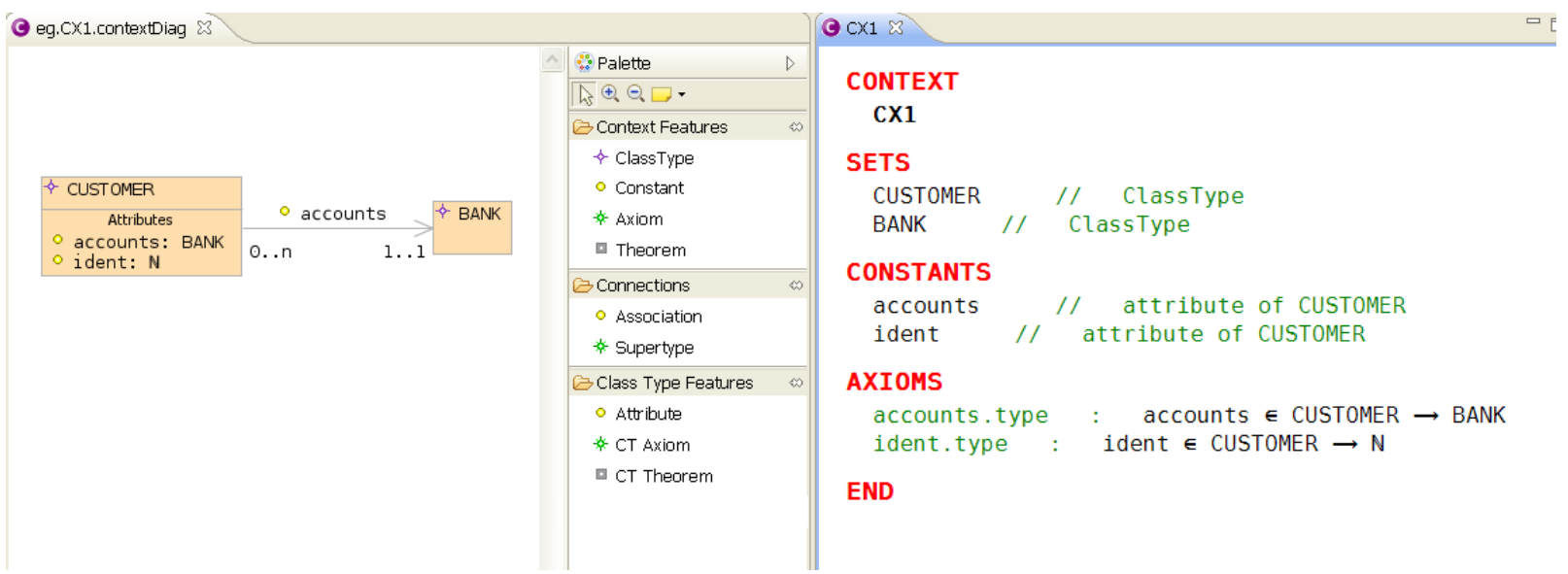

Fig. 2 Examples of Context Diagram and Event-B Translation

case the predicate must be true for all instances of the classtype. Classtypes are used to define types and also to define constant attributes of those types. The attribute ident and the association accounts are translated as constants. Each UML-B context gives rise to an Event-B context (i.e., the UML-B tool generates a corresponding Event-B context). Fig. 2 also shows the automatically generated Event-B for this example. Each Event-B statement is preceded by its label which defines its purpose. For example, ident.type is a label for the Event-B statement ident $\in$ CUSTOMER $\rightarrow \mathbb{N}$ that defines the type of the ident attribute.

Another kind of relationship between two classtypes, supertype (inverse subtype), is used to indicate that the source classtype is more specific than (a subset of) the target class type. Fig. 3 shows an example of subtyping Account into CurrentAccount and SavingAccount. In the generated Event-B machine, the supertype relationship will lead to the type of the classtypes CurrentAccount and SavingAccount being subsets of the instances of Account. I.e., CurrentAccount $\subseteq$ Account and SavingAccount $\subseteq$ Account. The sub-classtypes $C u r$ -

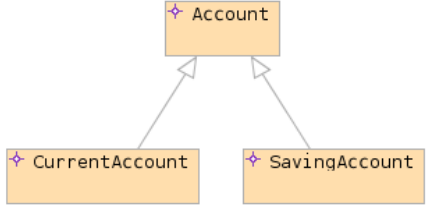

Fig. 3 Subtyping a Classtype in UML-B

rentAccount and SavingAccount give rise to constants in the generated Event-B context.

\subsection{Class Diagram}

A class diagram is used to describe the dynamic part of a model. A class diagram contains classes which represent fixed or variable subsets of the classtypes in a context diagram. Classtypes define the immutable fields (including associations) while classes define mutable fields. An object of a class can have both immutable and mutable fields since the class may be a subset of classtype. Like classtypes, each class may have 


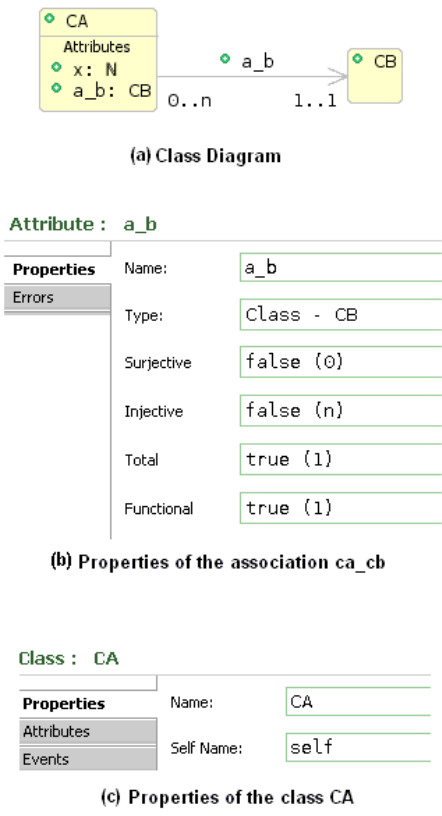

Fig. 4 Example of Class Diagram and class properties

attributes and associations. In addition, each class may own events and state machines. Class events and state machine transitions own actions that may modify the attributes and associations of any class. From each UML$B$ machine an Event-B machine is generated. The generated Event-B consists of variables generated from the classes, attributes, associations and state-machines and events generated from the class events and state-machine transitions. From each UML-B, we also generate an Event-B context to contain the sets and constants that are needed to define the classes and state-machines.

Similar to the supertype/subtype relationship between classtypes, a class may also subtype another class giving rise to similar subsets of instances but in this case the classes are represented by variables in the generated Event-B machine.

An example of a class diagram is given in Fig. 4(a) which consists of classes $C A$ and $C B$. In the generated Event-B implicit context these classes give rise to the sets $C A_{-} S E T$ and $C B_{-} S E T$ which are used as the base types (given, or carrier, sets) for the corresponding classes. (Note that a class may also subtype a classtype in which case these implicit base types are supressed). In the generated Event-B machine the classes give rise to variables. The class $C A$ contains an attribute $x$ of type $\mathbb{N}$ and an association $a_{-} b$ of type $C B$. The multiplicity property for the association $a_{-} b$ shown in Fig. 4(b) specifies a many-to-one relationship (i.e. total function). A full explanation of association multiplicity may be found in [11]. In the generated Event-

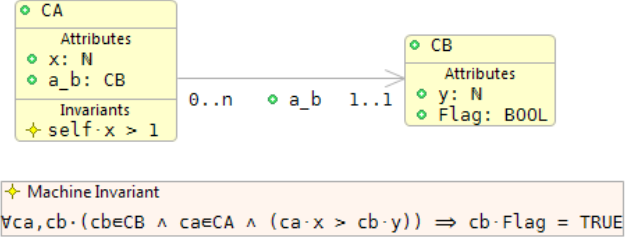

Fig. 5 Example of Class and Machine Invariants

B machine the attributes $x$ and $a_{-} b$ give rise to variables. For each class, attribute and association, a type invariant will be generated. For example, the class $C A$ is typed by an invariant which specifies that $C A$ is a subset of $C A \_S E T\left(C A \in \mathbb{P}\left(C A \_S E T\right)\right)$ and attribute $x$ is typed by the invariant $x \in C A \rightarrow \mathbb{N}$.

Each class has a self name property with the default value self, i.e., the identifier that is used to represent a contextual instance of a class in class events and invariants. This default may be overridden by the modeller by setting the self name property. The self name property of the class $C A$ is shown in Fig. 4(c). A class may have events whose parameters, guards and actions are defined explicitly as textual properties. Textual properties of a class, such as invariants, guards and actions, are written in the $\mu \mathrm{B}$ (micro $\mathrm{B}$ ) notation [11]. $\mu \mathrm{B}$ is identical to Event-B notation except that it uses an object-oriented style dot notation to navigate ownership of class entities such as attributes and associations. For example, i.x refers to the value of the variable $x$ which belongs to instance $i$.

A class may own invariants to express properties of its instances. For example in Figure 5, $C A$ has an invariant self. $x>1$ which says that all instances of $C A$ must have a positive $x$ value. The use of the class's self name implies universal quantification over the class instances. We may also place invariants in classes when they are not universally quantified over its instances, e.g. $\operatorname{card}(C A)>0$ in order to indicate their relevance to the class. We may optionally form machine invariants, which are not owned by any class. This is useful to express properties that are not applicable to any class or are equally applicable to several classes. For example, as shown in Figure 5, the machine invariant relates the class attributes $x$ of $C A$ and $y$ and Flag of $C B$.

\subsection{State Machine Diagram}

Fig. 6 shows an example of a state machine $S M$. The state machine is owned by the class $C A(6(\mathrm{a}))$. Fig. 6(b) shows its two states, $A$ and $B$ and the transitions $t 1$, t2, $t 3$ and $t 4$. The solid circle is the initial state and the solid circle with an outer circle is the final state. The 


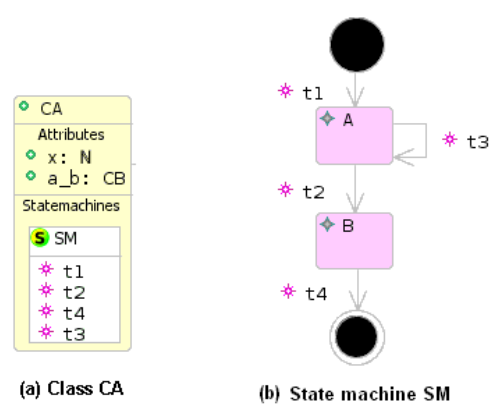

Fig. 6 Example of State Machine Diagram

translation to Event-B for a state machine can either be a state set representation or state function representation. UML-B allows modellers to switch between these two representations. For the state set representation, each state is represented by a variable which is a disjoint subset of the class instances, $C A$ :

$$
\begin{aligned}
& A \in \mathbb{P}(C A) \\
& B \in \mathbb{P}(C A) \\
& A \cap B=\varnothing
\end{aligned}
$$

That is, variable $A$ represents the set of instances of $C A$ that are in the state $A$ and similarly for $B$. For a state function representation, a single variable $S M$ represents the state-machine as a function mapping each instance of $C A$ to an enumeration of the set of states SM_STATES as follows:

$$
\begin{gathered}
S M_{-} S T A T E S=\{A, B\} \\
S M \in C A \longrightarrow S M_{-} S T A T E S
\end{gathered}
$$

In this paper, the translation to Event-B is described using the state set representation. The generated EventB machine for $M 1$ is shown in the Rodin screenshot, Fig. 7. Each Event-B statement is preceded by its label which describes its purpose. For example, CA.type is a label for the Event-B statement $C A \in \mathbb{P}\left(C A \_S E T\right)$. The states $A$ and $B$ of state machine $S M$ are represented by variable subsets of $C A$ which are disjoint (i.e. $A \cap B=\varnothing)$. An instance of $C A$ changes its state when a transition fires. For each transition there is a guard that specifies that the class instance is in the source state (labelled as ...isin_..) and actions that specify its entry to the target state (labeled as ...enterState_..) and its departure from the source state (labelled as .._leaveState_..). The parameter self is generated to refer to a non-deterministically selected contextual instance of the class $C A$. A transition from an initial state such as $t 1$, defines a constructor for the class. The translation of $t 1$ selects an unused instance and adds it to the set of $C A$ (labelled self.type). A transition to a final state such as $t_{4}$ is a destructor which removes an instance from the current instances and from the domain of all the class variables. The transition $t 3$ is a self loop transition which does not change state. Hence, in the generated Event-B the event $t 3$ has a guard that specifies its source state but no actions to change state.

Invariants and theorems can be attached to classes and or states and are automatically quantified over the class instances or adorned with an antecedent representing the containing state, as appropriate. A full explanation and examples of these is given in [1].

\subsection{Semantics of UML-B}

The semantics of UML-B models are given by the generated Event B model and Event-B semantics are defined by the corresponding proof obligations. Hence the semantics of UML-B can be deduced from [1] and [7]. In this section we give an intuitive semantics which should provide sufficient background for this paper.

Event-B models consist of variables and events. Event-B events are considered to be spontaneous, atomic guarded actions. When the guard of an event is satisfied, the event is enabled and may perform its actions in a single atomic substitution. Several different variables may be altered in parallel during this substitution. An event may have no guards, in which case it is always enabled, or no actions, in which case it does not alter the state of the variables. If one or more events are enabled one of them will fire. Events do not fire in parallel; if several events are enabled, one of them will be selected non-deterministically to fire and this may change the enabledness of the events. Notice that there is no construct to specify a sequence of events. The feasibility of a sequence of events is only determined by the individual guards and actions of those events. Typically, to specify a sequence of events, a modeller will introduce a variable that resembles a 'program counter' and devise appropriate guards and actions throughout the events to organise them into a sequence. As there is no conditional operator in Event-B, decisions are typically modelled using several alternative events. Within such a group of events, the guards are used to provide the condition for each alternative.

UML-B provides an alternative way to model Event$B$ variables and events. The constructs in UML-B that define data are classes, attributes, associations and statemachines. These data constructs provide additional structure to the types of the variables but in other senses are just Event-B variables. The constructs in UML-B that define events are class events and statemachine transitions. Class events are 'lifted' to a set of instances in an object-oriented manner and transitions impose sequencing (effectively by generating a 'program counter'). Transitions may also be 'lifted' if their state 


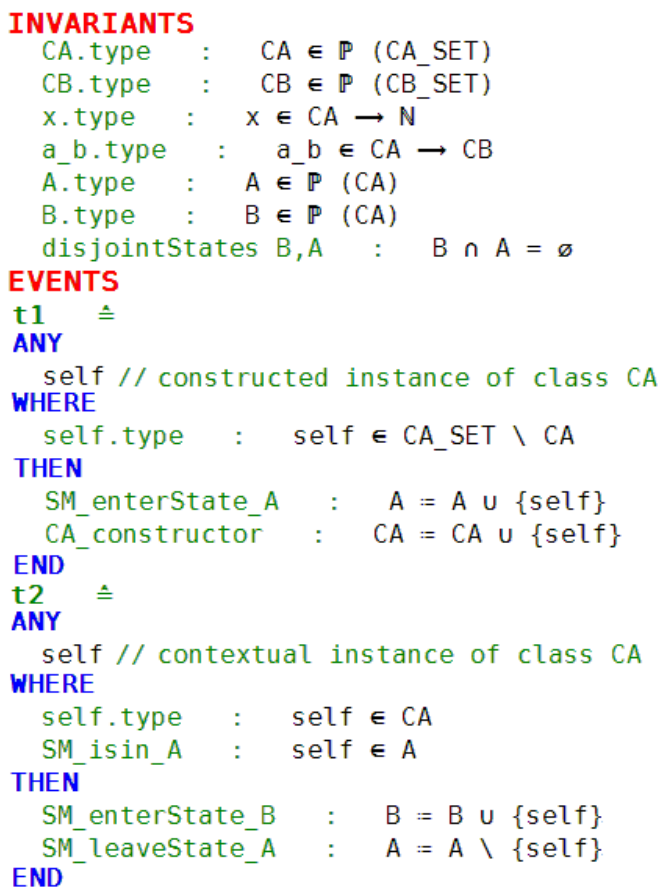

Fig. 7 Generated Event-B specification of M1

machine is owned by a class. As in Event-B groups of class events or groups of parallel transitions may be used to represent conditional execution. However, apart from these convenient additions, class events and transitions are just Event-B events. Therefore, like Event-B, UML-B semantics are based on the underlying concept of spontaneous atomic guarded actions that change the state of the variables.

Comparing with other commonly used semantics such as UML, UML-B has no concept of external events that may trigger transitions, no mechanism whereby one transition may invoke another and, as there is no so-called 'big-step', terms such as 'run to completion' have no meaning. All these things can be, and often are, modelled explicitly when required by constructing suitable control variables and guards.

\subsection{UML-B model transformation workflow}

Despite its name, UML-B is an entirely independent notation from the UML. We suggest that it is 'UMLlike' and will feel familiar to UML users, however, it has its own meta-model and no UML models are involved in our discussions here. In [33] we discuss a case study where a UML model of a railway interlocking was translated by hand into UML-B for verification purposes. There are currently no tools that automatically translate UML-B models to or from UML.

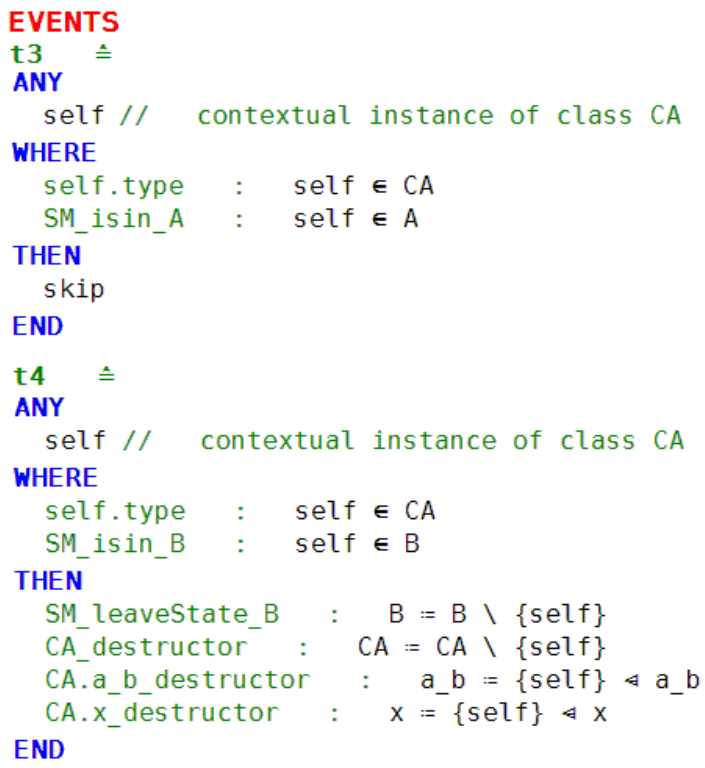

Initial versions of UML-B were implemented as extensions to UML using the UML profile mechanism. However, UML is a very rich notation compared with our target language, Event-B, and many features of UML are redundant to our purpose. On the other hand, even where a UML feature seems applicable, Event-B imposes a particular semantics which is often slightly at odds with that of UML. We found that the combination of unused features and 'false-friends' caused confusion and hence we decided to implement UML-B as an independent notation with its own meta-model.

The translation from UML-B model to Event-B is performed programmatically in two steps. Firstly by a translation to an internal representation of the Event$\mathrm{B}$ model and then by programmatic generation of the final Event-B model via the Rodin API. Both steps are performed by 'hand-coded' Java programs and the internal representation of Event-B is represented by 'hand-coded' Java classes. Since this implementation was produced, improvements in meta-model transformation technologies such as QVT [35] and the provision of an EMF framework for Event-B [34] would facilitate an improved model transformation approach.

\subsection{UML-B meta-model}

The UML-B meta-model [1] defines the abstract syntax of the UML-B language. The UML-B meta-model 
is described using the ecore notation from the Eclipse Modelling Framework (EMF) [29]. The ecore notation is based on the OMG's Meta Object Facility (MOF) which is a subset of the UML class diagram notation. Generalisation is used extensively to ensure that common attributes of UML-B model elements are defined in a way that promotes generic, reflective tooling. The meta-model is an exact description of the abstract syntax of the UML-B language and is used to automatically generate repository and editing utility code using the EMF technology. The Eclipse Modelling Framework is a framework and code generation facility for building applications based on a meta-model. Another Eclipse framework, the Graphical Modelling Framework (GMF) [30], is used to automatically generate the code for the UML-B graphical diagram editor.

To give a flavour of the UML-B meta-model we show a small part of it in Fig. 8. There are three kinds of relationships used between meta-classes in the metamodel: generalisation, reference and containment. An example of a generalisation (a link with a large triangular arrowhead) is from UMLBPredicate to UMLBelement indicating that a $U M L B P r e d i c a t e$ is a specialisation of the meta-class UMLBelement. This means that a UMLBPredicate can be treated as a UMLBelement and includes its properties. An example of a reference (a link with a small arrowhead) is the refines link from $U M L B$ Machine to itself which specifies that a machine may refine at most one other machine but may be refined by many machines. An example of a containment (a link with a solid diamond at the source end) is the classtypes link from UMLBContext to UMLBClassType indicating that a context may contain many classtypes. The metaclass UMLBelement is a base meta-class that provides a name property and an error marking system for recording modelling errors, for all sub-typed model elements. UMLBconstrainedElement, which sub-types UMLBelement, provides a base meta-class for elements that own constraints (axioms or invariants) and theorems which are elements of another subtype, UMLBPredicate. UMLBPredicate owns a string property, predicate, to contain the text of the predicate. UMLBMachine and UMLBContext are subtypes of $U M L B C o n s t r u c t$ and hence indirectly UMLBconstrainedElement so that they can own such constraints. A UMLBMachine can also own a collection, classes of UMLBClass and a UMLBContext can own a collection classtypes of UMLBClassType. Fig. 8 shows a small part of the UML-B meta-model and omits many features such as state-machines, variables and events.

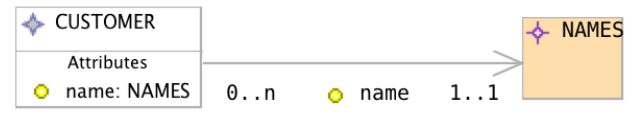

Fig. 9 Example of Extended Context Diagram

\section{Overview of Refinement in UML-B}

We first give an intuitive overview of refinement in UMLB by explaining how it relates to Event-B refinement. Since UML-B is based upon the underlying formalism provided by Event-B, so is its notion of refinement. The refinement techniques that are available in UML-B are those of Event-B but the extra structuring provided by UML-B's higher level modelling constructs are reflected in its concepts of refinement. We have previously [25] introduced class diagram refinement and state machine refinement but not context diagram extension. Here, we provide a more complete overview to scope the available features and refinement choices in UML-B.

\subsection{Data Refinement in UML-B}

In Event-B context extension, sets and constants are always retained and may be added to. Hence, in UMLB, class-types may be extended with new features (attributes, associations, axioms and theorems) but we do not need to specify which old features, or even which old class-types, are retained; they all are. We need to be able to refer to the old class-types in order to extend them with new features. In diagrammatic modelling terms, we need a graphic representation of the old class-type as a container for new features. Only the new features represent part of the refined model; the container is a skeleton to provide contextual information that forms part of the definition of the new features. If we do not want to add any new features to a class-type we can omit the skeleton from the refined model.

For example, referring back to the example in Fig. 1 and Fig. 2, in the extended context $C X 2$ we might add an association name from the extended classtype $C U S$ TOMER to a new classtype NAMES as shown in Fig. 9. Notice that we do not need to repeat anything about the previously defined attributes of CUSTOMER or the classtype $B A N K$ since these are still accessible from $C X 1$. The only purpose of the extended classtype CUSTOMER is to assist in defining the type of the new association.

Variables, on the other hand, may be discarded in Event-B refinement so that they can be refined by new 


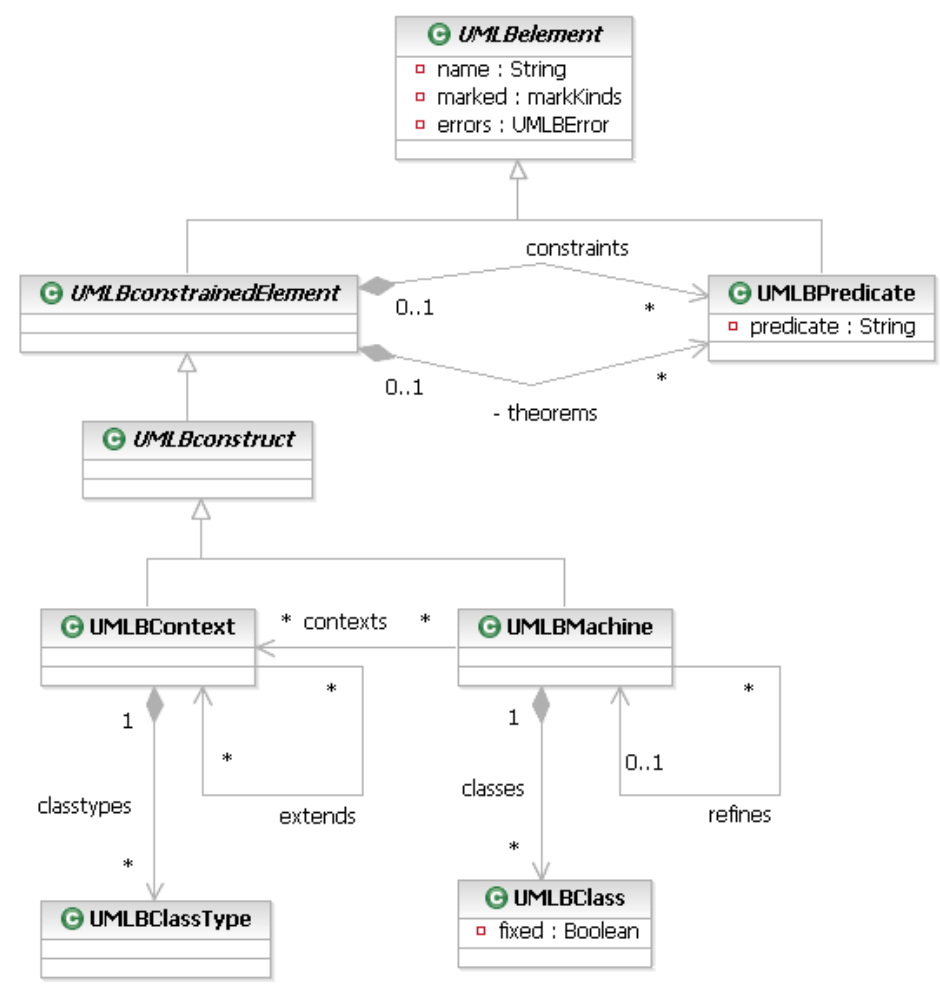

Fig. 8 UML-B meta-model (part of)

data of a different name and possibly a different type. Refinement relations are captured by specifying invariant properties, called gluing invariants, that relate the corresponding values of new and old variables in the refined model. Variables may be retained by repeating their name in the refinement. Hence in UML-B refinement, not only do we need refined classes as skeleton containers in order to refine features of the class, but also to indicate that the variable representing the instances of the class is to be retained. In this case, unless we wish to refine the class with some other data variable, we cannot omit the skeleton even if we do not wish to alter its contained features. (UML-B also allows classes to have a fixed set of instances in which case their skeletons are similar to class-types since their instances are defined as a constant in a context).

In addition, since class attributes, associations and state-machines represent variables we need to indicate which of these are to be retained. We do this using inherited attributes and refined state-machines. The former merely defines that the attribute or association is to be retained whereas the latter also acts as a container for any nested state-machines that are added in the refinement. These retained data features must remain contained in the same classes as their abstract counterparts in order to preserve their types and re- fined state-machines must contain the same states as their abstract counterparts.

The following schematic illustrates class refinement in terms of attributes.

\section{Class C \\ a1 \\ a2}

In the refinement, class $C$ inherits attribute $a 1$ and drops attribute $a 2$, which is refined by a new attribute $a 3$, and $C$ also has a new attribute $a 4$. A gluing invariant is needed to relate the dropped attribute to those that replace it. For example a2 could be a boolean abstraction of a threshold which is detailed in a3. Hence the gluing invariant might be:

$$
\forall c \cdot c \in C \Rightarrow(a 3(c) \geq \mathrm{T} \Leftrightarrow a 2(c)=\mathrm{TRUE})
$$

Apart from these considerations, the rules of data variable refinement in UML-B machines are quite flexible in that we may discard any of the previously defined variable data structures (classes, attributes, associations or state-machines) and replace them with new ones which may be of a different kind. To do so, we must provide a gluing invariant so that the verifier can establish that the refined events have an equivalent behaviour to the abstract events that they refine. 


\subsection{Event Refinement in UML-B}

Event-B events may be refined by retention, renaming or splitting. When refining a retained or renamed event, parameters may be added or replaced provided that the equivalent of any removed parameter is demonstrated in a witness predicate, guard conjuncts may be added or replaced as long as the overall guard is not weakened, new actions may be added as long as they only modify new variables and existing actions may be replaced provided they behave in an equivalent way, according to the data refinement. Splitting is a special case where a group of events, representing different conditional cases, all refine the same abstract event that did not reveal the individual cases. New events may be added as long as they only alter new variables. New events are often added as preliminary steps leading up to a refined event.

In UML-B, class events can be refined with equal flexibility. A class event can simply be retained and refined by adding to or replacing its parameters, guards and actions to reflect the data refinements of the class diagram, or the event may be renamed or split into several cases. The requirement to preserve containment observed for class data features does not apply to class events. Since event containment merely defines a parameterisation of the event, we can move events to different classes as long as we provide a witness to demonstrate an equivalent for the lost class instance parameter. The containment of an event in a particular class is chosen for convenience so that a class parameter is automatically generated and guards and actions are automatically lifted to that instance. The same event can always be specified by placing it outside of the class and manually adding the class instance parameter, adjusting the guards and actions accordingly.

The following schematic illustrates the refinement options for five events e1, e2, e3, e4 and e5, which are initially all contained in class $C$.

\section{Refined Class C}

e1 (refines e1)

e6 (refines e2)

e3a (refines e3)

e3b (refines e3)

\section{Class D}

$$
\begin{aligned}
& \text { e4 (refines e4) } \\
& \quad \text { witness: }(\text { selfC }=\text { selfD.assoc })
\end{aligned}
$$

No Class (machine level event)

$$
\begin{aligned}
& \text { e5 (refines e } 5) \\
& \quad \text { parameter: }(p \in \mathbf{C})
\end{aligned}
$$

witness: $($ self $C=p)$

The event $e 1$ remains in class $C$ and merely refines its abstract version whereas $e \mathscr{2}$ has been renamed $e 6$. Event $e 3$ has been split into two cases $e 3 a$ and $e 3 b$ which both refine $e 3$. In this example they both remain in class $C$ but it is also possible to move either or both at the same time as splitting. Event $e 4$ has been moved to another class $D$. In doing so, it loses the implicit self parameter (provided by the UML-B to Event-B translation) for the contextual instance of $C$. To satisfy the refinement we need to provide a replacement and specify its equivalence via a witness. In this example we assume some association assoc from $D$ to $C$ which can provide an instance of $\mathrm{C}$. Note that all guards and actions would need to be rewritten to take account of the parameter change. Event $e 5$ has been moved to machine level (i.e. not contained in any class). Here again we need to provide a replacement for the lost implicit self parameter. In this example it is provided by a new parameter which is an instance of class $C$.

The transitions in state-machines also represent Event-B events. It is possible to refine UML-B transitions into UML-B events by providing a data refinement that replaces the state-machine's states with some other data that provides an equivalent model of state. We might wish to do this when approaching an implementation if the state-machine view is considered to be an abstract representation of some concrete system variables. For example, a transition soundAlarm with source state highTemp could be refined to an event with guard temperature $>$ limit under the data refinement state $=$ highTemp $\Leftrightarrow$ temperature $>$ limit

Usually, however, we do not refine away our statemachines but build them up through refinement into more elaborate models of a system where the statemachine represents a central 'mode-based' organisation of the system's behaviour. Transition refinement is just as flexible as class event refinement except with respect to its state-machine. That is, as with class events, we can retain, rename and split transitions, refining their behaviour to reflect data refinements but we cannot change the transition's source state since this would not preserve the abstract guard and we cannot change its target state since this would not preserve the abstract behaviour. The only thing we can do to transitions diagrammatically is to split them into two or more cases with the same source and target states. However, we can refine state-machines using a particular kind of data refinement where we break down a state into several sub-states by nesting a new state-machine inside the parent state. This allows us to reveal more detailed behaviour in the form of extra transitions (representing 


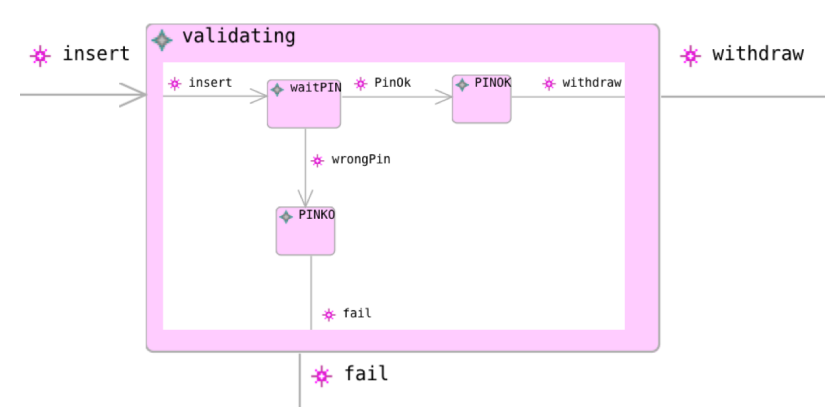

Fig. 10 Example of superposition refinement by state machine nesting

new events) between those sub-states as well as more detailed targets and sources (strengthened guards and refined actions) for the incoming and outgoing transitions, respectively, of the parent state. As a consequence, splitting of transitions in the parent state machine is often necessary when separate cases of the original transition are revealed by the additional detail introduced in the nested state machine

In summary, a state machine may be refined via two complimentary techniques:

- A transition may be replaced by several transitions representing different sub cases of the original transition.

- A state may be elaborated by a nested state machine adding more detailed behavour.

Fig. 10 shows an example where a state validating is refined by a nested state machine that adds details concerning pin number validation. However, this is a manufactured illustration. In the current version of the UML-B tool, nested state machines are modelled in separate diagrams from their parent state and a transition elaboration property is needed to link transitions in a nested state machine to the corresponding incoming and outgoing transitions of the super-state. In a nested state machine, a transition from an initial state elaborates exactly one incoming transition to the super-state and a transition to a final state elaborates exactly one outgoing transition from the super-state.

\section{Formalisation of Rules of Refinement in UML-B}

In this section, we provide a formalisation of the syntactic rules of refinement in UML-B. We do this using the Event-B notation. We limit ourselves to a description of the aspects that are particular to UML-B and do not cover those features which are a direct reflection of the corresponding Event-B rules. For a formalisation of
Event-B refinement see Abrial's treatment of Event-B [7]. The formalisation is presented in two sections, class diagram refinement and state machine refinement. We introduce a base set ELEMENT to represent all UML$\mathrm{B}$ elements and then partition this into subsets to represent the distinct kinds of elements.

\section{Partition(ELEMENT,CLASSES,ATTRIBUTES, EVENTS,STATEMACHINES,STATES, TRANSITIONS)}

We refer to the UML-B machine to be refined as M1 and the resulting refined machine as M2.

\subsection{Formal Definition of Class Diagram Refinement}

We define specific collections, $C 1, A 1, E 1$ and $S M 1$ of the element types to represent the class diagram of $M 1$.

$$
\begin{gathered}
C 1 \subseteq C L A S S E S \\
A 1 \subseteq A T T R I B U T E S \\
E 1 \subseteq E V E N T S \\
\text { SM1 } \subseteq \text { STATEMACHINES }
\end{gathered}
$$

We represent the containment of attributes, events and state machines by their owning classes as functions.

$$
\begin{aligned}
\text { containmentA1 } & \in A 1 \rightarrow C 1 \\
\text { containmentE1 } & \in E 1 \rightarrow C 1 \\
\text { containmentSM1 } & \in S M 1 \rightarrow C 1
\end{aligned}
$$

We define the components of M2, representing the result of refinement of $M 1$, in a similar fashion and with corresponding constraints resulting in C2, A2, E2, SM2, containmentA2, containmentE2, containmentSM2.

Now that we have defined $M 1$ and $M 2$, we represent the changes made in going from M1 to M2. Let $R E M_{-} C 1$ be the subset of $C 1$ classes which are removed and $N E W_{-} C L$ be the set of new classes added in the refinement.

$$
\begin{gathered}
R E M_{-} C 1 \subseteq C 1 \\
N E W_{-} C L \subseteq C L A S S E S \backslash C 1
\end{gathered}
$$

Similarly for attributes, events and state machines.

$$
\begin{gathered}
R E M_{-} A 1 \subseteq A 1 \\
N E W_{-} A T \subseteq A T T R I B U T E S \backslash A 1 \\
R E M_{-} E 1 \subseteq E 1 \\
N E W_{-} E V \subseteq E V E N T S \backslash E 1 \\
R E M \_S M 1 \subseteq S M 1
\end{gathered}
$$




$$
N E W_{-} S M \subseteq S T A T E M A C H I N E S \backslash S M 1
$$

Let containmentNE $W_{-} A T T$ and containmentNE $W_{-} S M$ represent the containment of new attributes and new state machines in classes of $M 2$ respectively.

$$
\begin{aligned}
& \text { containment NE } W_{-} A T T \in N E W_{-} A T \rightarrow C 2 \\
& \text { containment } N E W_{-} S M \in N E W_{-} S M \rightarrow C 2
\end{aligned}
$$

The rules defining the elements of the refined class diagram in machine $M^{2}$ are as follows:

Rule C1 - Classes of M2: The classes of M2 consists of the classes of $M 1$ excluding the removed classes and adding the new classes:

$$
C 2=\left(C 1 \backslash R E M_{-} C 1\right) \cup N E W_{-} C L
$$

Rule C2 - Attributes of M2: The attributes of M2 consists of the attributes of $M 1$ excluding the removed attributes and adding the new attributes:

$$
A \mathscr{2}=\left(A 1 \backslash R E M_{-} A 1\right) \cup N E W_{-} A T
$$

Rule C3 - Containment of the attributes of M2: The containment of attributes in $M 2$ is the same as that of $M 1$ omitting the removed attributes and adding the containment of the new attributes: ${ }^{2}$

$$
\begin{gathered}
\text { containment } A \mathscr{2}=\left(R E M \_A 1 \& \text { containment } A 1\right) \cup \\
\text { containment } N E W_{-} A T T
\end{gathered}
$$

Rule C4 - Events of M2: The events of M2 consists of the events of $M 1$ excluding the removed events and adding the new events:

$$
E 2=\left(E 1 \backslash R E M_{-} E 1\right) \cup N E W_{-} E V
$$

Rule C5 - State machines of M2: The state machines of M2 consists of the state machines of $M 1$ excluding the removed state machines and adding the new state machines:

$$
S M 2=\left(S M 1 \backslash R E M \_S M 1\right) \cup N E W_{-} S M
$$

Rule C6 - Containment of the state machines of M2: The containment of state machines in M2 is the same as that of $M 1$ omitting the removed state machines and adding the containment of the new state machines:

$$
\begin{gathered}
\text { containmentSM2 }=\left(R E M_{-} S M 1 \triangleleft \text { containmentSM1 }\right) \\
\cup \text { containmentNE } W_{-} S M
\end{gathered}
$$

Notice that we do not introduce any rules about the containment of events in M2. This is because events can be freely moved between classes. Instead we need to define the refinement relationships between the events in $M 2$ and those in $M 1$.

\footnotetext{
2 Where $S \& r$ removes a set $S$ from the domain of a relation $r$ (domain subtraction).
}

$$
\text { evRefinementE2 } \in \text { E2 } \rightarrow \text { E1 }
$$

This is a partial function since some events in E2 may represent superimposed behaviour and not refine any abstract event (sometimes referred to as 'refining skip'). We also require that every retained event refines itself

$$
\forall e \cdot e \in E 1 \backslash R E M \_E 1 \Rightarrow \text { evRefinementE2 }(e)=e
$$

and every removed event is refined by at least one new event.

$$
\begin{gathered}
\forall r \cdot r \in R E M_{-} E 1 \Rightarrow \\
\exists n \cdot n \in N E W_{-} E V \wedge \text { evRefinementE2 }(n)=r
\end{gathered}
$$

Furthermore, for every event $e$ that has moved to a different class, i.e. containmentE1 $(e) \neq$ containmentE2(e), we need to add a witness predicate

$$
W(c a, C 2, A 2, c c)
$$

with,

$$
\begin{aligned}
& c a \in \text { instances }(\text { containmentE1 }(e)) \\
& c c \in \text { instances }(\text { containmentE2 }(e))
\end{aligned}
$$

Utilising the classes C2 and attributes A2 of M2, the witness predicate $W$ establishes a relationship between the disappearing parameter $c a$, representing the contextual class instance of the abstract version of the event, and the new parameter $c c$, representing the contextual class instance of the concrete event.

The final stage is to add sufficient invariants concerning $C 1, A 1, C 2, A 2$ (and any ancillary variables used) to enable the simulation proofs of refinement. The process of discovering these invariants is discussed in section 7. These steps correspond with Event-B as covered in chapter 5 of [7].

\subsection{Formal Definition of State Machine Refinement}

We define specific collections to represent the state-machine diagrams of $M 1$

$$
\begin{gathered}
S 1 \subseteq S T A T E S \\
T 1 \subseteq T R A N S I T I O N S
\end{gathered}
$$

and represent containment of these states and transitions via functions that map each to its containing state-machine.

$$
\begin{aligned}
& \text { containmentS1 } \in S 1 \rightarrow S M 1 \\
& \text { containmentT1 } \in T 1 \rightarrow S M 1
\end{aligned}
$$

We also represent the relationship between the transitions and their source and target states with functions.

$$
\text { sourceState } T 1 \in T 1 \rightarrow S 1
$$




$$
\text { targetState } T 1 \in T 1 \rightarrow S 1
$$

The source and target of a transition must be contained in the same state-machine as the transition.

$$
\forall t \cdot t \in T 1 \Rightarrow
$$

containmentS1 $($ sourceState $T 1(t))=$ containment $T 1(t)$

$$
\wedge
$$

containmentS1 $($ targetState $T 1(t))=$ containment $T 1(t)$

We represent the transition elaborates relationship, distinguishing between those that elaborate an outgoing transition of the super-state from those that elaborate an incoming transition.

$$
\begin{aligned}
& \text { elaborateOutgoing } T 1 \in T 1 \longmapsto T 1 \\
& \text { elaborateIncoming } T 1 \in T 1 \longmapsto T 1
\end{aligned}
$$

Note that these functions are injective because each elaborating transition can elaborate at most one parent transition. They are partial because the domains contain both incoming and outgoing elaborating transitions. If an elaborating transition elaborates both an incoming and an outgoing transition, the super-state transition is a self loop. This is the only case where the domains of the functions intersect.

$$
\forall t \cdot t \in
$$

$\operatorname{dom}($ elaborateOutgoing $T 1) \cap \operatorname{dom}($ elaborateIncoming 11

$$
\Rightarrow \text { elaborateOutgoing } T 1(t)=\text { elaborateIncoming } T 1(t)
$$

We define the components of $M 2$, representing the result of refinement of $M 1$, in a similar fashion and with corresponding constraints resulting in S2, T2, containmentS2, containmentT2, sourceStateT2, targetStateT2, elaborateOutgoing T2, elaborateIncomingT2. We define the refinement relationships between the transitions in T2 and those in T1.

$$
\text { trRefinementT2 } \in \text { T2 } \rightarrow \text { T1 }
$$

This is a partial function since elaborating transitions contribute to other transitions and do not directly represent events. Also, depending on the type of refinement, T2 may contain new transitions which do not have a refines relationship.

Now that we have defined the components of $M 1$ and M2, we represent the changes made in going from M1 to M2. In UML-B refinement, the structure of a refined state machine is an elaboration of the structure of its abstraction in two possible ways:

1. Transitions may be split into several transitions with the same source and target states.

2. States may be elaborated by a nested state machine.

First we describe the requirements for (1). For clarity, we describe a refinement where only one transition is refined. In practice, it is possible to split several transitions in one refinement step and add nested statemachines at the same time, however this is equivalent to making a series of simple refinements as described here. Let $t r$ be a transition of $T 1$ which is to be replaced by a set of new transitions Ttr in the refinement.

$$
\operatorname{tr} \in T 1
$$

$$
T t r \subseteq T R A N S I T I O N \backslash T 1
$$

Rule T1: States of M2. The states of $M 2$ and their containment are unchanged by this refinement.

$$
S \mathscr{2}=S 1 \wedge \text { containmentS2=containmentS1 }
$$

Rule T2: Transitions of $\mathbf{M 2}$. The transitions of M2 are the transitions of $M 1$ with $t r$ replaced by Ttr.

$$
T \mathscr{2}=(T 1 \backslash\{t r\}) \cup T t r
$$

Rule T3: Containment of the transitions of M2. The containment of transitions in M2 is the same as that of $M 1$ except that the new transitions replace $t r$ and all have the same container as $t r$.

$$
\begin{gathered}
\text { containmentT2 }=(\{t r\} \varangle \text { containment } 1) \cup \\
(\operatorname{Ttr} \times\{\text { containmentT } 1(t r)\})
\end{gathered}
$$

Rule T4: Source and target states of M2 transitions. The source/target states of transitions in M2 are the same as that of $M 1$ except that the new transitions replace $t r$ and all have the same source/target state as $t r$.

$$
\begin{gathered}
\text { sourceState T2 }=(\{t r\} \& \text { sourceState T1 }) \cup \\
(\text { Ttr } \times(\text { sourceStateT1 }(\text { tr })\}) \\
\text { targetStateT2 }=(\{\text { tr }\} \text { targetStateT1 }) \cup \\
(\text { Ttr } \times(\text { targetStateT1 }(\text { tr })\})
\end{gathered}
$$

Rule T5: Refinement relationship of M2 transitions. In this step we have not added any new transitions; the 'new' transitions of Ttr are actually different cases of $t r$. Therefore, every transition of Ttr refines the original transition $t r$ and every other nonelaborating transition is unchanged and hence refines itself.

$$
\begin{gathered}
\operatorname{dom}(\text { trRefinementT2 })=\text { T2 } \\
(\operatorname{dom}(\text { elaborateOutgoing T2 }) \cup \\
\operatorname{dom}(\text { elaborateIncomingT2 })) \\
\forall t \cdot t \in T t r \Rightarrow \operatorname{trRefinementT2~}(t)=t r \\
\forall t \cdot t \in \operatorname{dom}(\text { trRefinementT2 }) \backslash T t r \Rightarrow \\
\text { trRefinementT2 }(t)=t
\end{gathered}
$$


Next we describe the requirements for (2) where, in a refinement, a nested state machine is added into a state of M1. For clarity, we describe a refinement where only one state is refined. In practice, it is possible to combine this with other refinements including refining several states in one refinement step. However this is equivalent to making a series of single state refinements.

Let $s t \in S 1$ be the state which is being refined. Let $I N_{-} T s t$ be the set of all incoming transitions into the state st, i.e., those transitions of $T 1$ whose target state is $s t$ but whose source state is not.

$$
\begin{aligned}
I N_{-} T s t= & \{t \mid t \in \mathrm{T} 1 \wedge \text { targetState } T 1(t)=\text { st } \wedge \\
& \text { sourceState } T 1(t) \neq \text { st }\}
\end{aligned}
$$

Let $O U T_{-} T s t$ be the set of all outgoing transitions from the state st, i.e., those transitions of $T 1$ whose source state is st and whose target state is not.

$$
\begin{aligned}
\text { OUT_Tst }= & \{t \mid t \in T 1 \wedge \text { sourceState } T 1(t)=\text { st } \wedge \\
& \text { targetState } T 1(t) \neq \text { st }\}
\end{aligned}
$$

Let $L O O P_{-} T s t$ be the set of all looping transitions from the state st, i.e., those transitions of $T 1$ whose source and target states are both st.

$$
\begin{gathered}
\text { LOOP_Tst }=\{t \mid t \in T 1 \wedge \text { sourceState } T 1(t)=s t \wedge \\
\text { targetState } T 1(t)=\text { st }\}
\end{gathered}
$$

Let $s m$ be the new nested state machine to be added to st and let $S s m$ and Tsm be its new sets of states and transitions respectively.

$$
\begin{gathered}
s m \in S T A T E M A C H I N E S \backslash S M 1 \\
\text { Ssm } \subseteq \text { STATES } \backslash S 1 \\
T s m \subseteq \text { TRANSITIONS } \backslash T 1
\end{gathered}
$$

Let sourceStateTsm and targetStateTsm map each new transition to its source and target state respectively.

$$
\begin{aligned}
& \text { sourceStateTsm } \in T s m \rightarrow S s m \\
& \text { targetStateTsm } \in T s m \rightarrow S s m
\end{aligned}
$$

The new transitions are partitioned into initial, final, internal elaborating and internal non-elaborating transitions.

$$
\begin{gathered}
\operatorname{partition}\left(T s m, I N I_{-} T s m, F I N_{-} T s m, I N T_{-} E L A B_{-} T s m,\right. \\
\text { NON_ELAB_Tsm) }
\end{gathered}
$$

We now define one-to one mappings (injections) to represent the elaborates relationship of the new state-machine. Each initial transition of $s m$ elaborates one incoming parent transition of st, each final transition elaborates an outgoing parent transition and each internal elaborating transition elaborates a parent loop transition.

$$
\begin{aligned}
& \text { elaborateIncomingTsm } \in I N I_{-} T s m \longmapsto I N_{-} T s t \\
& \text { elaborateOutgoingTsm } \in \text { FIN_Tsm } \nrightarrow O U T \_T s t \\
& \text { elaborateLoopTsm } \in \text { INT_ELAB_Tsm } \nrightarrow L O O P_{-} T s t
\end{aligned}
$$

Rule S1: States of M2. M2 has all the states of M1 as well as the states of the new state-machine $s m$.

$$
S 2=S 1 \cup S s m
$$

Rule S2: Containment of the states of M2. The containment of states in M2 is the same as that of $M 1$ but adding the containment of the new states in sm.

$$
\text { containmentS2 }=\text { containmentS1 } \cup(S s m \times\{s m\})
$$

Rule S3: Transitions of M2. M2 has all the transitions of $M 1$ as well as the transitions of the new statemachine $s m$.

$$
\text { T2 }=T 1 \cup T s m
$$

Rule S4: Containment of the transitions of M2. The containment of transitions in M2 is the same as that of $M 1$ but adding the containment of the new transitions in $s m$.

$$
\text { containmentT2 }=\text { containmentT1 } \cup(T s m \times\{\mathrm{sm}\})
$$

Rule S5: Source states of $M 2$ transitions. The source state mapping of the transitions in M2 is the same as that of $M 1$ but adding the source state mapping of the new transitions in $s m$.

$$
\text { sourceState T2 }=\text { sourceStateT1 } \cup \text { sourceStateTsm }
$$

Rule S6: Target states of $M 2$ transitions. The target state mapping of the transitions in M2 is the same as that of $M 1$ but adding the target state mapping of the new transitions in $s m$.

$$
\text { targetState } T_{2}=\text { targetStateT1 } \cup \text { targetStateTsm }
$$

Rule S7: Outgoing elaborating transitions of M2. The outgoing elaborations of the transitions in M2 is the same as that of $M 1$ but adding the outgoing and loop elaborations of the new transitions in $\mathrm{sm}$.

$$
\begin{gathered}
\text { elaborateOutgoing T2 = elaborateOutgoing T1 } \cup \\
\text { elaborateOutgoingTsm } \cup \text { elaborateLoopTsm }
\end{gathered}
$$

Rule S8: Incoming elaborating transitions of M2. The outgoing elaborations of the transitions in M2 is the same as that of $M 1$ but adding the outgoing and loop elaborations of the new transitions in $\mathrm{sm}$.

$$
\begin{gathered}
\text { elaborateIncoming T2 = elaborateIncomingT1 } \cup \\
\text { elaborateIncomingTsm } \cup \text { elaborateLoopTsm }
\end{gathered}
$$


Rule S9: Refinement relationship of M2 transitions. The transitions Tsm are new and therefore do not have refinement relationships. Every other nonelaborating transition in T2 is unchanged and hence refines itself.

$$
\begin{gathered}
\operatorname{dom}(\text { trRefinementT2 })=\text { T2 } \backslash(\text { Tsm } \cup \\
\operatorname{dom}(\text { elaborateOutgoingT2 }) \cup \\
\operatorname{dom}(\text { elaborateIncomingT2 })) \\
\forall t \cdot t \in \operatorname{dom}(\text { trRefinementT2 }) \Rightarrow \\
\text { trRefinementT2 }(t)=t
\end{gathered}
$$

\section{Enhancement of the UML-B Meta-model to support Refinement}

This section describes enhancements to the UML-B meta-model which were required to support refinement of UML-B models. We refer to the version of UML-B before the extensions as UML-B Version 1 and after the extension as UML-B Version 2. (UML-B Version 1 corresponds to reference [1]. UML-B Version 2 was extended and used for, but not reported in, reference [25]). Version 1 already contained some features that are needed for refinement. These were a refines reference feature from UMLBMachine to itself to support machine refinement, a refines reference feature from $U M L$ BguardedAction to itself to support event and transition refinement relationships (UMLBguardedAction being a super type for both UMLBEvent and UMLBTransition) and an extends reference feature from $U M L$ BContext to itself to support context extension. The support for refinement of model elements that represent data was entirely missing from Version 1 and required the introduction of new meta-classes to represent data that had already been introduced in previous refinement levels. The support for refinement of model elements that represent events is simpler because this can be achieved by introducing new event elements that reference the abstract ones. Indeed, this is how EventB manages event refinement. Support for event refinement was already present in Version 1 apart from one minor addition that was needed to support refinement of state-machine transitions.

\subsection{Support for Refinement of Class Diagrams}

In UML-B Version 1 there was no mechanism to distinguish a class that was being refined from a newly defined class. Although a class could be retained by repeating it in the refined class diagram, the EventB generation would produce invariants to re-define the variables representing the class instances at each refinement level leading to overcomplicated Event-B. Similarly there was no way to distinguish attributes and associations that were being retained in the refined class from those that were newly introduced. The Event-B generation would reproduce invariants to re-define old attributes and associations at each refinement level. To provide better support for refinement of classes a new meta-class, UMLBRefinedClass, was introduced to represent the 'skeleton' of a refined class (Fig. 11). This, and the meta-class for new classes UMLBClass, both subtype a common ancestor UMLBabstractClass which provides the type for the containment of classes in a machine. UMLBRefinedClass has none of the properties of UMLBClass, such as name, super-type etc., since a refined class should not be able to re-define these properties. When such properties are needed by the refined class, for example to display a label on the class diagram or to generate the Event-B representation of a contained attribute, they must be obtained from the original class that has been refined. Therefore, the only property possessed by the meta-class UMLBRefinedClass is a refines reference. The target of this reference is of type UMLBabstractClass to allow for a chain of several refinement levels. I.e. the target of the refines reference may, itself, be a refined class.

A similar arrangement was also introduced to support attributes that are to be retained in a refined class. In this case we refer to them as 'inherited' attributes since they cannot be refined in any sense. The new meta-classes are UMLBabstractAttribute and UMLBInheritedAttribute, the latter having a reference inherits targeting the former and the former being the target type for the containment of attributes in classes. Note that UML-B associations are an alternative concrete syntax for UML-B attributes, hence no separate meta-model arrangement is required for inherited associations.

\subsection{Support for Extension of Context Diagrams}

In UML-B Version 1 there was no mechanism to distinguish a classtype that was being extended with new features from a newly defined classtype. If the modeller repeated the class type in order to extend it, the Event$\mathrm{B}$ generation would produce constants and axioms to re-define the class type in the context extension, leading to Event-B errors. A pattern of meta-classes similar to that used for classes and attributes was introduced to support extension of classtypes. The new meta-classes are UMLBabstractClassType and UMLBExtendedClassType, the latter having a reference extends targeting the former and the former being the target type 


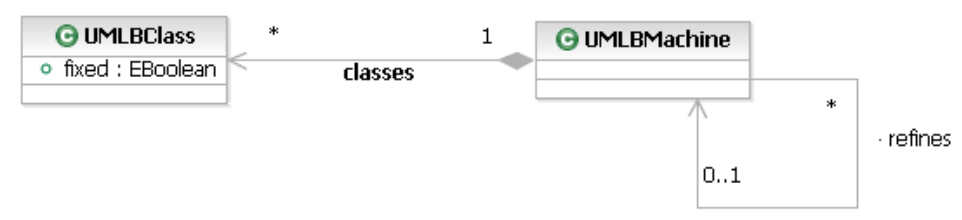

(a) Previous metamodel

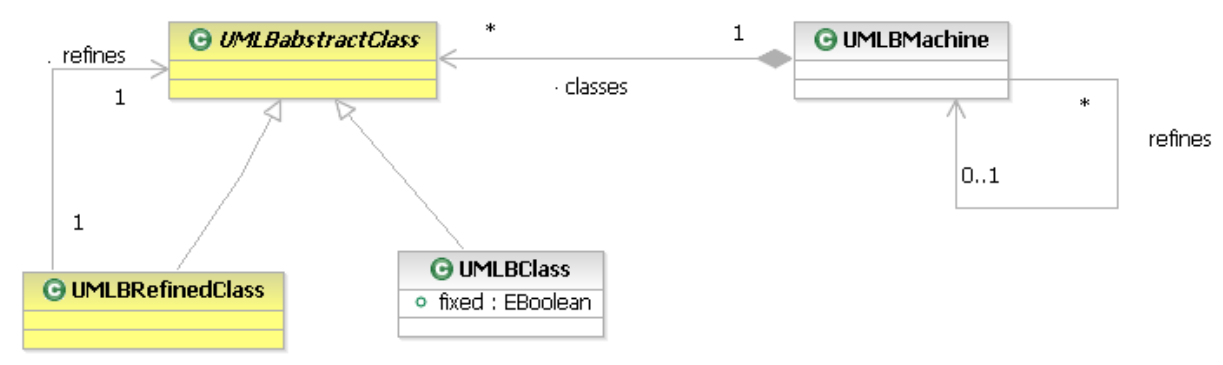

(b) Changes to the previous metamodel

Fig. 11 UML-B meta-model enhancements to support the refinement of classes

for the containment of class types in contexts. Note that nothing is needed for class type attributes and class type associations because they are always visible through extensions and therefore do not need to be retained in an extension.

\subsection{Support for Refinement of State-machine Diagrams}

In UML-B Version 1 there was no mechanism to distinguish a state-machine that was being refined from a newly defined state-machine. If the modeller repeated the state-machine in order to refine it, the Event-B generation would repeat data and constraints to represent the state-machine state in the new refinement level leading to Event-B errors. A pattern of meta-classes similar to that used for classes and attributes was applied both for the refinement of state-machines and for the refinement of states resulting in the new metaclasses UMLBabstractStatemachine, UMLBRefinedStatemachine, UMLBabstractState and UMLBRefinedState. Some features of state-machines, such as name, are owned by UMLBStatemachine and therefore not available to UMLBRefinedStatemachine and must be obtained via the refines relationship, whereas some features of state-machines are owned by UMLBabstractStatemachine so that they are also available for redefinition in a refined state-machine. Most notably, a refined state-machine owns its own set of transitions so that transition refinement can be undertaken. The containment of states is also moved to UMLBabstractStatemachine however, unlike transitions, this is a consequence of the need to create new refined states at each refinement level rather than a modelling facility.

At this point we note that we have used the same meta-model pattern for all of the UML-B elements that represent Event-B data (class, class type, attribute, state-machine and state). The generic pattern may be described as the containment of instances of an abstract super type which are partitioned into actual model elements and 'skeleton' model elements, the latter having a reference relationship to an instance of the abstract super-type which should eventually, via transitive closure, provide an actual model element.

There is one final enhancement to the UML-B metamodel which was introduced in UML-B Version 2. It concerns the relationships between the newly introduced transitions of a nested state-machine and those incoming and outgoing transitions connected to its parent state. The nested state-machine contains some initial and final transitions which contribute to existing events that have, in the previous level, already been generated by the incoming and outgoing transitions of the parent state. For these initial and final transitions we need to specify which parent transition they contribute to so that the generation can add their guards and actions to the corresponding existing event. Similarly, internal transitions may contribute to self-looping tran- 
sitions of the parent state. To provide the reference between the nested transition and the parent transition two new references, elaborates and its inverse $i s E$ laboratedBy were added to the meta-model as a selfreference on the meta-class UMLBTransition.

\section{Overview of ATM Case Study in UML-B}

This section presents the development of an ATM case study in UML-B using refinement. The development uses all the extensions of UML-B meta-model.

The package diagram in Fig. 12 shows the contexts, the five levels of machines and their relationships where a machine sees a context, a context extends another context and a machine refines another machine. The summary of the five machine levels are given here.

Abstract machine (ATM_A): The abstract machine models the accounts in a bank and a number of operations that may be performed on the accounts.

First Refinement (ATM_R1): The first refinement introduces a set of ATMs as a medium to withdraw money or to check an account balance.

Second Refinement (ATM_R2): The second refinement introduces a concept of PIN number and models an explicit validation for cards.

Third Refinement (ATM_R3): The third refinement introduces the request and response communication between an ATM and the bank and splits a withdrawal into a bank transition and an ATM transition.

Fourth Refinement (ATM_R4): The fourth refinement models the send and receive events of the request and response communication between ATMs and the bank. This is done by adding a receive event for each request and adding a send event for each response. The send event for request refines the abstract request event. The receive event for response refines the abstract response event. The fourth refinement also introduces a set of requesting ATMs whose requests are being processed by the bank.

We outline some class and state machine diagrams of the ATM case study referring to the refinement rules in Section 4. Details of the development in UML-B can be found in [25]. Fig. 13 shows some of the class diagrams of the ATM case study. The machine ATM_A consists of a class account (Fig. 13(a)) with its attribute bal and four events namely, createAccount, deposit, withdraw and checkBalance. The account class represents the set of accounts that currently exist in the system. The attribute bal represents the balance of an account.

The class diagram of $A T M_{-} R 1$ (Fig. 13(b)) contains the new class atm and a refined class account that refines the account class of ATM_A. These two element of classes in $A T M_{-} R 1$ referred to refinement Rule $C 1$. The class atm has three attributes which are atm_acbal, $a t m_{-} c a s h$ and $a t m_{-} c a r d$. The attribute atm_acbal represents an account balance after each withdraw cash or check balance transaction via an ATM. The attribute atm_cash represents a stock of cash in an ATM. The attribute atm_card represents a card in an ATM. The refined class account inherits the bal attribute. These refinement elements refers to Rule C2 and C3. The refined class account refines the two events, namely, createAccount and deposit of the abstract account class of machine ATM_A. The other two events of its abstract class namely, withdraw and checkBalance are moved to the new class $\mathrm{atm}$ in this refinement level as transitions in the state machine $A T M_{-} S M$ of the class atm. At the abstract level, we specify the effect of a withdrawal on the account balance. In the refinement, we further specify that the withdrawal takes place via an ATM. At the abstract level it is natural to specify the withdrawal as an event of the account class while in the refinement it is natural to specify it as a transition of the $\mathrm{atm}$ class. The events element refers to Rule $C_{4}$, where withdraw and checkBalance events are removed and no new event is added. The refinement rules referred are Rule $C 5$ and C6 when adding the state machine $A T M_{-} S M$. The transitions of the state machine are explained later in this section.

The class diagram (Fig. 13(c)) of ATM_R2 contains the two refined classes that refine the account and $\mathrm{atm}$ classes of $A T M_{-} R 1$ machine. The refined class atm of $A T M_{-} R 2$ contains the refined state machine $A T M_{-} S M$.

Fig. 14 shows some state-machine diagrams of the ATM case study. The state-machine ATM_SM in Fig. 14(a) partitions the behaviour of an ATM into either an idle state, (i.e., not being used/not active) or active_atm state (i.e., is being used). If a transition $t 1$ is triggered and the current state is the source state of t1, the ATM changes state. The transition start creates an instance of ATM and adds it to the set atm_card, initialises its stock of cash as MAX_CASH and changes its state to idle. The insertCard transition can be triggered when an ATM is in the idle state and the inserted card is a valid ATM card. When it is triggered it changes the ATM state from idle to active_atm. The reloadCash transition can trigger when an ATM is in the idle state and the ATM cash amount is less than the $M A X_{-} C A S H$. The reloadCash transition will top up the ATM cash to the maximum amount $M A X_{-} C A S H$. The ejectCard transition changes an ATM state from active_atm to idle and removes the ATM from the set atm_card. While an ATM is in active_atm state, it means, an ATM user can use it for withdrawal or checking an account balance (i.e., checkBalance transition). The withdrawOK 


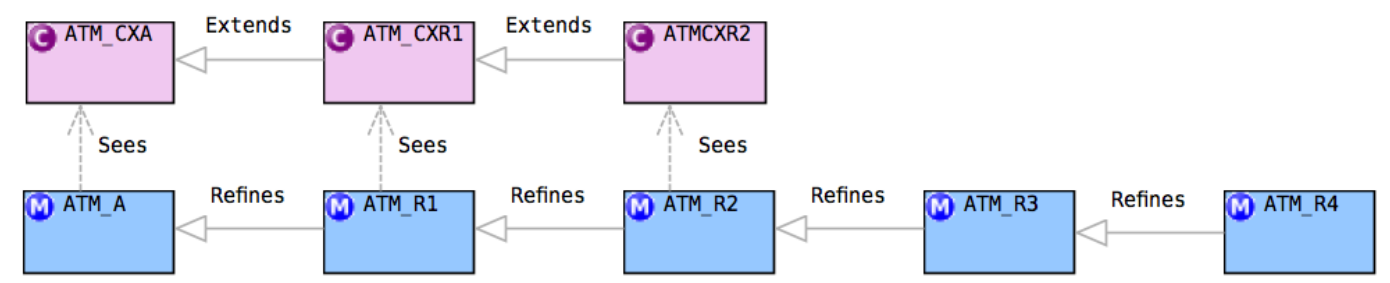

Fig. 12 ATM Package Diagram

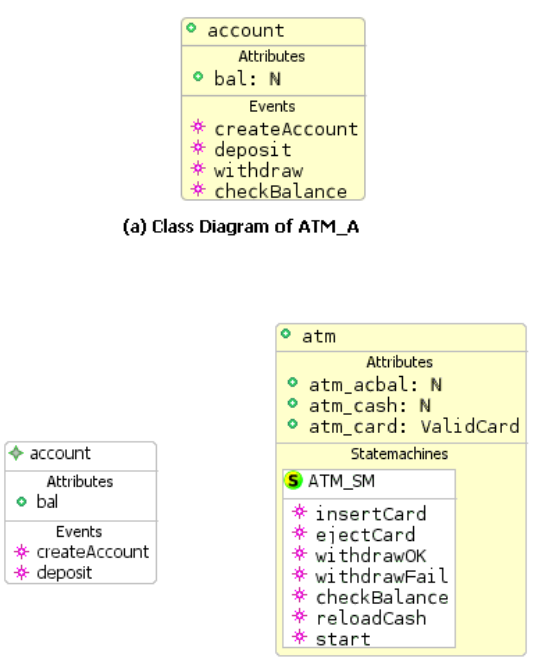

(b) Class Diagram of ATM_R1

Fig. 13 Class diagrams of ATM

transition represents a successful withdrawal transaction, whereas, the withdrawFail transition represents a failure possibly because the withdrawal amount exceeds the account balance.

The refined class atm of $A T M_{-} R 2$ contains the refined state-machine $A T M_{-} S M$ (Fig. 14(b)) which contains the two refined states that refine the states idle and active_atm of the state machine ATM_SM of $A T M_{-}$R1. The transition ejectCard is split into three transitions namely ejectCard1, ejectCard2 and ejectCard3 which refine ejectCard. The other five transitions refine themselves. This refinement refers to Rules T1, T2, T3, T4 and T5. These rules assumed that the state active_atm does not have state machine active_atm_SM yet. For Rule T1, the states of $A T M_{-} R 2$ and their containment are the same as ATM_R1. Referring Rule T2, ejectCard1, ejectCard2 and ejectCard3 replaced ejectCard. As in Rule T3, the container of the replacing transitions are the same as ejectCard's. Similarly, for their source and target states as in to Rule T4. The refinement relationship refers Rule $T 5$.
The state machine active_atm_SM of $A T M_{-} R 2$ is like the one in Fig. 14(c) but without nested state machines in states transOption and performTrans. When the state machine active_atm_SM is added, referring to Rule $S 1$, the states of $A T M_{-} R 2$ are extended to include new states validating, invalidCard, transOption and perform Trans. Rule S2 defined the containment of all the states. The new states contained in the state machine active_atm_SM. For Rule S3, new transitions consists of initial elaborating, final elaborating, internal elaborating and internal non-elaborating. Initial elaborating is insertCard. Final elaborating are ejectCard1, ejectCard2 and ejectCard3. Internal elaborating are withdrawOK, withdrawFail and checkBalance. Internal nonelaborating are validateCardOK, validateCardFail, retry and doAnother. As in Rule $S_{4}$, the container of new transitions is the state machine active_atm_SM. As in Rule $S 5$ and $S 6$, all new transitions must have their source and target states. Referring Rule $S \%$, the outgoing elaborate relationships include the new final elaborating and internal elaborating transitions with respective outgoing transition of super-state active_atm. For 


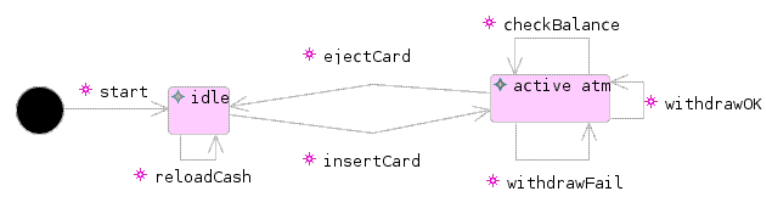

(a) State machine ATM_SM of class ATM of machine ATM_R1

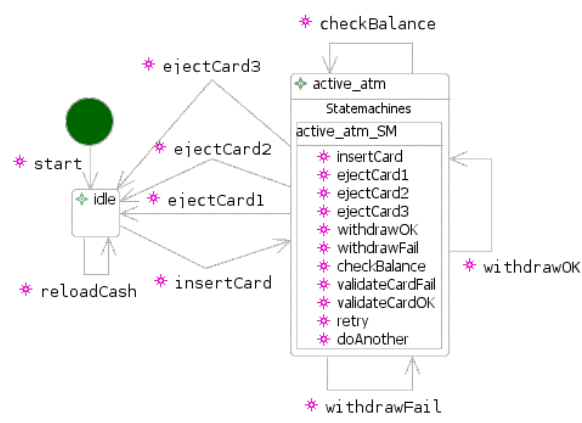

(b) Refined State machine ATM_SM of Refined Class ATM of machine ATM_R

Fig. 14 State Machine Diagrams

Rule $S 8$, the incoming elaborate relationships include the new initial final elaborating and internal elaborating transitions with respective incoming transition of active_atm. Referring Rule S9, all non-elaborating old transitions refines themselves.

Fig. $14(\mathrm{c})$ is a refined state machine active_atm_SM of machine $A T M_{-} R 3$ which shows that the refined state transOption and the refined state performTrans have nested state machines. This approach of elaborating states with sub-states in refinement supports an incremental refinement approach. The ATM case study has shown that the extensions of the meta-model and drawing tools are working as expected.

The ATM case study reported in this paper differs slightly from the one presented in [25] although they are based on the same version of the UML-B meta-model. The differences are:

- In [25], we did not have classtypes of ATM, Card and Pin to give rise to the sets ATM, Card and Pin in an Event-B context. Instead the sets are generated from the classes in the class diagram and contained in the Event-B implicit context. Thus, there were no context diagrams to be extended with new classtypes. In the case study, we wanted the ability to extend the classtypes in refinements to introduce immutable attributes. Therefore, for the ATM model in this paper, we created a UML-B context diagram containing the three classtypes which were then used as instances for the classes.

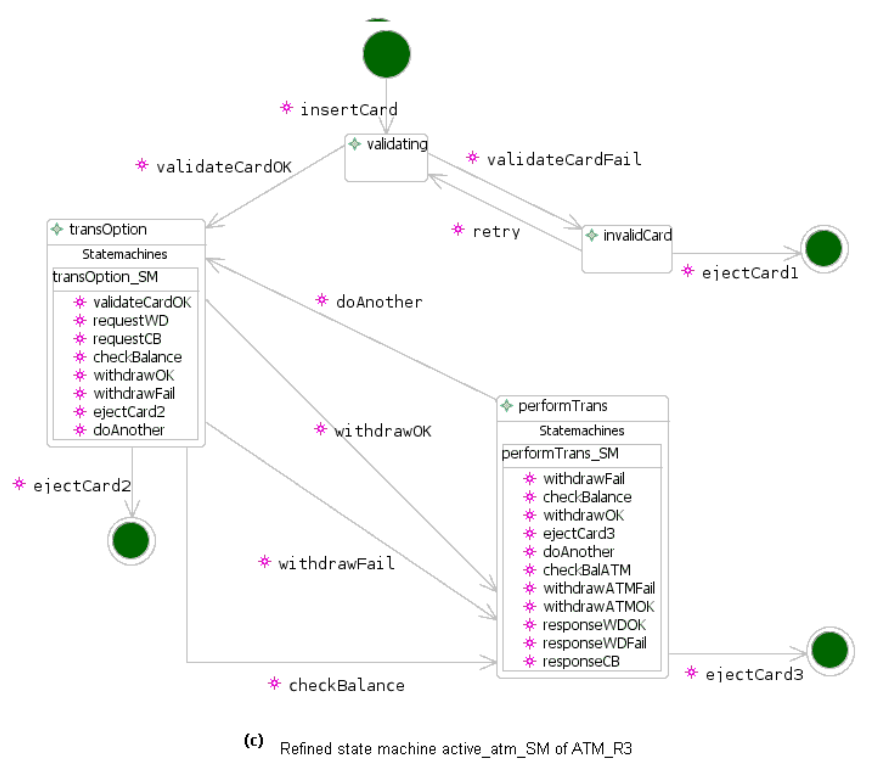

- The refinement strategy is slightly changed in this paper. In [25], the splitting of withdrawal into bank and ATM transitions is done in the second refinement. We think this is not reasonable because the transition withdrawal request that causes the withdrawal ATM transition is not introduced until the third refinement. It is more reasonable to delay introducing the withdrawal ATM transition until the third refinement. This improves the sequence of transitions between request and response. Ideally, the response should come when there exists a request. In this case, request $W D$ is the request made by the user via an ATM machine, while responseWDOK and responseWDFail are the responses to the user from the ATM. This improves the cohesiveness of the refinements and allows the second refinement to deal with pin validation.

- In this paper we have formalised the refinement rules and explicitly refer to them in the ATM case study.

An archive of the UML-B development of the ATM case study can be down-loaded. ${ }^{3}$ UML-B is a plug-in to the Rodin platform which can be downloaded. ${ }^{4}$ UML-B can then be installed from the update site contained in Rodin (Help-Install New Software: select 'Rodin' up-

\footnotetext{
3 ATM case study: http://eprints.soton.ac.uk/346101/

4 http://sourceforge.net/projects/ rodin-b-sharp/files/Core_Rodin_Platform/
} 
date site). Instructions on using Rodin (including installation of plug-ins) are available ${ }^{5}$.

\section{Proofs and Invariants}

This section discusses the proofs of the UML-B case study and also the construction of gluing invariants using Rodin provers.

All the proof obligations (POs) for the five machines of the ATM case study were generated and proved using the Rodin tool provers [5]. The statistics are outlined in Table 1 showing the total POs for each level (POs), the number of POs which are automatically discharged (aPOs) and the number of POs which are interactively discharged (iPOs).

In $A T M_{-} R 3$, there are seven interactively discharged POs. Three POs are discharged manually by proving that two related states are disjoint and another four are proved by rewriting the partition invariant into its definition. A similar way is used to prove the seven interactively POs in ATM_R4. Two POs are discharged by manually proving that two states are disjoint and the other five POs are discharged by rewriting the partition invariant.

Where refinements have been made a gluing invariant may be needed to relate the abstract data to the new data. In general, finding suitable invariants can be non-trivial and care must be taken not to introduce unnecessary invariants which can increase the proof burden as every event must be shown to preserve them. We discuss two alternative approaches to constructing gluing invariants. Firstly, by discovery from information provided by the prover and secondly, by design, from information in the model. Both methods are based on the refinement pattern of nesting state-machines but are not in any way specific to the ATM case study.

\subsection{Constructing a Gluing Invariant by Discovery}

Some of the gluing invariants are constructed by using guidance from the undischarged proof obligations. We describe first our method of discovering the gluing invariants. Then we give some examples of discovering the invariants for the ATM case study.

In this paragraph we describe our method for discovering the gluing invariants. We inspect an undischarged PO , $\mathrm{H} \vdash \mathrm{G}$, (consisting of some available hypotheses $\mathrm{H}$ and a goal $\mathrm{G}$ ) and construct an invariant of the form $\forall x \cdot H^{\prime} \Rightarrow G$ where $H^{\prime}$ is a subset of the list of hypotheses $H$ and $x$ represents the list of free variables

\footnotetext{
5 http://handbook.event-b.org/current/html/ tut_install_plugins.html
}

that correspond to event parameters. The selection of hypotheses $h$ from $H$ to appear in $H^{\prime}$ is based on these rules:

1. $h$ is of the form $p \in S$, where $p$ is an event parameter and $S$ represents a state of a state machine. In particular $S$ is the sub-state of a nested state machine.

2 . The free variables of $h$ are included in the free variables of $G$

In the next paragraphs, we describe some examples of discovering invariants using the above rules (1) and (2). One of the discovered gluing invariants is in the third refinement $\left(A T M_{-} R 3\right)$. An attempt to construct the invariant is done by using the interactive prover. The $A T M_{-} R 3$ was run in a proving perspective without having any gluing invariant which results in a number of undischarged proof obligations. The first undischarged PO is given here as an example. The prover cannot discharge the guard atm_cash $($ self $A T M) \geq a m$ of the event withdrawOK. The hypotheses and the goal are as follows:

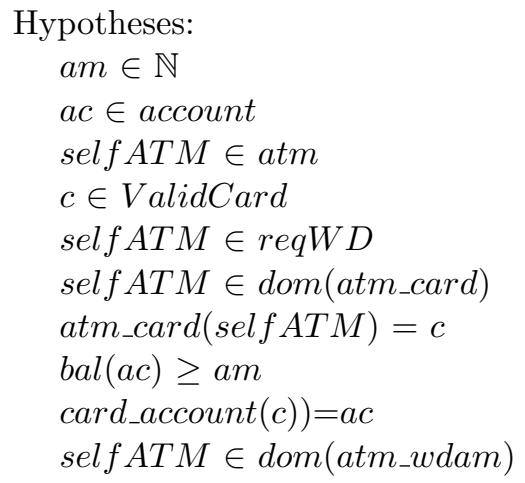

The goal:

$a t m_{-} c a s h(\operatorname{self} A T M) \geq a t m_{-} w \operatorname{dam}(\operatorname{self} A T M)$

From the above PO, the prover is trying to prove that the cash in an ATM is greater or equal to a given withdrawal amount. This is true for any successful cash withdrawal. According to rule (1), selfATM is the event parameter concerned in the goal and req $W D$ is a substate of a nested state machine performTrans_SM (Fig. 14 (c)). Therefore, from the list of hypotheses, self $A T M \in$ reqWD is selected as one of the hypotheses in the gluing invariant. Also, atm_wdam is the free variable included in the goal. Thus, according to rule (2), self ATM $\in$ dom(atm_wdam) is also selected as the hypotheses in the gluing invariant. The required invariant is represented in Event-B as follows:

$\forall \operatorname{self} A T M \cdot \operatorname{self} A T M \in \operatorname{req} W D \wedge$

self $A T M \in$ dom (atm_wdam)

$\Rightarrow a t m \_c a s h(\operatorname{self} A T M) \geq a t m \_w d a m(\operatorname{self} A T M)$

Another example is the gluing invariant in the fourth refinement $\left(A T M_{-} R_{4}\right)$. The prover cannot discharge the 


\begin{tabular}{|c|c|c|c|}
\hline Machines & POs & aPOs & iPOs \\
\hline ATM_A & 4 & 4 & 0 \\
\hline ATM_R1 & 47 & 47 & 0 \\
\hline ATM_R2 & 68 & 68 & 0 \\
\hline ATM_R3 & 167 & 160 & 7 \\
\hline ATM_R4 & 149 & 142 & 7 \\
\hline Total & 435 & 421 & 14 \\
\hline
\end{tabular}

Table 1 Statistics from the Proof Effort

guard selfATM $\in$ dom(atm_card) of the event withdrawOK. The hypotheses and the goal are as follows:

Hypotheses:

$$
\begin{aligned}
& \text { self } A T M \in \text { atm } \\
& \text { self } A T M \in \text { recvdReqWD } \\
& \text { self } A T M \in \text { atmB } \\
& \text { self } A T M \in \text { dom }\left(a t m \_w d a m B\right) \\
& \text { atm_cardB(selfATM })=c \\
& \text { am }=\text { atm_wdamB }(\text { self } A T M) \\
& \text { card_account }\left(a t m \_c a r d B(\text { self } A T M)\right)=a c
\end{aligned}
$$

The goal:

$$
\text { self } A T M \in \text { dom(atm_card) }
$$

The prover is trying to prove that a given ATM has an ATM card in it. Similar to the first example, following rule (1), selfATM is the event parameter concerns in the goal and recvdReqWD is the sub-state of the nested state machine reqWD_SM of the sub-state reqWD. Thus, self $A T M \in$ recvdReqWD is selected forming the gluing invariant. The required invariant is represented in Event-B as follows:

$\forall$ self $A T M \cdot$ self $A T M \in$ recvdReqWD $\Rightarrow \operatorname{self} A T M \in d o m\left(a t m \_c a r d\right)$

The task of finding gluing invariant is also the same for the undischarged PO involving the guard self ATM $\in \operatorname{dom}\left(a t m \_c a r d\right)$ of the event checkBalance, i.e., when selfATM is in the sub-state recvdReqCB. The two invariants can be combined forming a single invariant as:

$\forall$ self $A T M \cdot$ self $A T M \in($ recvdReqWD $\cup$ recvdReqCB $\Rightarrow$ self $A T M \in \operatorname{dom}($ atm_card)

Another example of finding the gluing invariant in the fourth refinement is when the prover cannot discharge the guard atm_card $($ self $A T M)=c$ of the event withdrawOK. The hypotheses are the same as the first example of $A T M_{-} R 4$ and the goal is as follows:

$$
a t m \_c a r d(\text { self } A T M)=a t m \_c a r d B(\operatorname{self} A T M)
$$

Similarly, from the PO, using rules (1) and (2), the discovered gluing invariant is as follows:

$\forall$ self $A T M \cdot$ self $A T M \in$ recvdReqWD $\wedge$

self $A T M \in d o m\left(a t m_{-}\right.$cardB $)$

$\Rightarrow a t m_{-} c a r d($ self $A T M)=a t m_{-}$cardB
However, these rules are heuristics and they do not provide a complete method for verifying refinements. But they were sufficient to prove the refinements in our ATM development.

We would like to point out that UML-B is not a purely graphical notation. In particular we need to use a textual representation of gluing invariants in order to prove the refinement. All the discovered invariants are specified in UML-B as invariants in class diagrams.

\subsection{Constructing a Gluing Invariant by Design}

While it is attractive to let the prover indicate the invariants it needs, and to have a heuristic for constructing them mechanistically, it is also possible to construct sufficient gluing invariants purposefully. This can either be done before running the prover, or using the proof obligation goal as a hint. The latter differs from the invariant discovery method in that the gluing invariant is constructed by examining the model rather than examining the goal and hypotheses; the goal is only used as a hint of what to look for in the model.

An abstract model has many possible valid refinements but when modelling we choose one particular refinement and wish to verify that it is correct. The prover can verify that it is a refinement but it cannot tell whether it is the particular refinement we intended unless we provide some extra information. By expressing the linkages between the abstract model and the refined one, a gluing invariant indicates 'why' it is a refinement and hence indicates 'which' refinement was intended. If we let the prover tell us which gluing invariant to use we lose this extra verification condition and there is a danger we will end up with a verified but wrong system. In practice the likelihood of constructing a valid but wrong refinement may be remote, particularly as we get the opportunity to examine the gluing invariants thrown up by the prover since the discovery method is not fully automatic. However, there is some motivation at least for a more constructive approach to gluing invariants so that the modeller is forced to understand the refinement more intimately. 


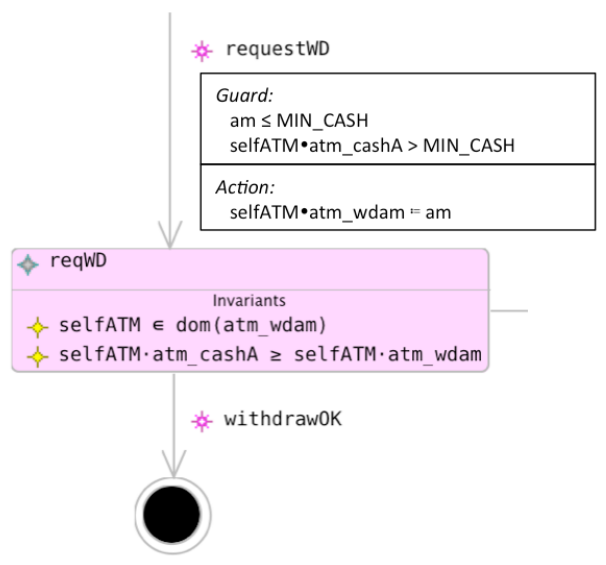

Fig. 15 Gluing Invariants by Design

Therefore as an alternative approach to invariant discovery, we show how invariants can be chosen by design using the state-machine refinement structure as a guide. This simply consists of placing invariants inside the new states introduced in a state-machine refinement. The gluing invariant is generally located in the new state which has outgoing final transitions that elaborate an old transition. The incoming transitions represent new preliminary steps leading up to this refined transition. However it may be necessary to add invariants in other new states where a sequence of new transitions is involved. Placing the invariant inside the state implies that it is true only while in that state and UML-B automatically adds an appropriate antecedent (corresponding to those chosen by heuristics in the discovery method) to this effect. The invariant is chosen by looking at the guards and actions of the incoming transitions to find properties that are true for all incoming transitions. There are two cases; state invariant properties may be true because the incoming transition is only taken when the property is true (and the actions do not change it) or because the transition establishes the property via its actions. Notice that, in the first case, such unchanged properties might not be explicitly mentioned in the guards if they are implied by the source state guard. Therefore, such properties may need to be carried forward from a previous state to the next state. Since the state invariants are derived from the guards and actions of the incoming transitions they are certainly true and (usually) easily proved. Certainly any transitions other than the incoming ones will be easily proved since they will clearly negate the antecedent and since the invariants are derived in a simple way from the incoming transitions, there is also a good chance that the automatic provers will find appropriate hypotheses easily. Hence it is not a problem to be quite liberal in

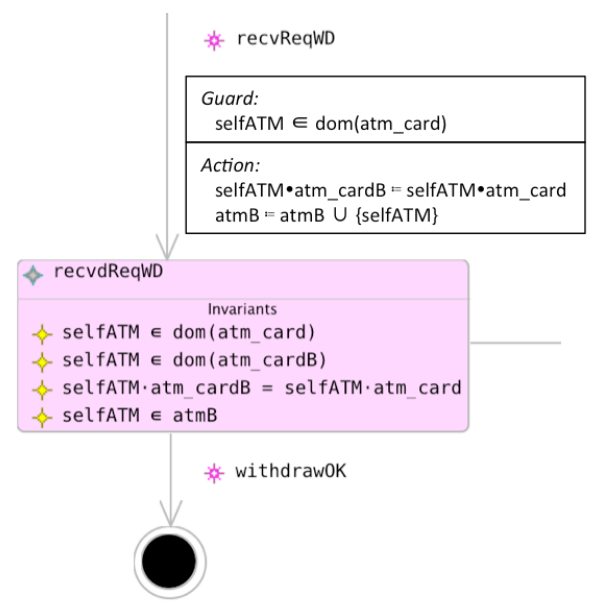

adding these invariants. If we have now defined everything relevant about the source state of the subsequent outgoing refined transition, there should be sufficient information for the proof of the refinement (otherwise the model must be faulty). The prover easily finds these hypotheses because it contains an instance of the antecedent quantification in its guard (corresponding to the generated source state guard). Of course this only works if there is no interference from other events or state-machines that may be in parallel with this one, but that is equally true of invariant discovery. (Such interference may indicate a complex gluing invariant that is not amenable to systematic methods or, more probably, that there is a mistake in the model). Adding these state invariants is quite easy if you understand the refinement and this is sufficient to allow the prover to prove the PO. Effectively, state invariants like this provide a link between the intermediate state spaces in a sequence of transitions which is exactly what the prover is lacking. They do this by linking our explicit generated annotation of states to the underlying conceptual state space.

As an example (Fig. 15) we show the gluing invariants constructed for the same event, withdrawOK, of $A T M_{-} R 3$ that featured in the first example of invariant discovery. This is a transition for which we have added some preliminary transitions request $W D$ within a new nested state-machine. Examining the guards and actions of request $W D$ we see that the variables of interest are atm_cashA and atm_wdam both parameterised for the instance selfATM. The parameter $a m$ is local and therefore cannot figure in the invariant. The action sets selfATM.atm_wdam to a value which is less than selfATM.atm_cashA hence the second invariant. We need to know that atm_wdam is a partial function (and have some experience of feasibility proof obliga- 
tions) to realise that selfATM.dom(atm_wdam) is important. There are other invariants that we could have derived involving $M I N_{-} C A S H$ which would have done no harm but turn out to be unnecessary. After adding these state invariants, which are directly equivalent to those added by the discovery method, the proof completed automatically. The second example corresponds to the same event, withdrawOK, of $A T M_{-} R_{4}$ that featured in another example of invariant discovery. Here, all the guards and actions are reflected as state invariants in a straightforward manner and again, this was sufficient to automatically discharge all the relevant proof obligations.

In the case of interference from another event, the invariants might be violated. To prevent the violation we may need to add a guard to the interfering event. For example, consider Fig. 15. If there was another event that was not part of the state machine of this figure that modifies selfATM.atm_cashA, then it could violate the invariant selfATM.atm_cashA $\geq$ selfATM.atm_wdam. An example would be an empty_cash event with the action selfATM.atm_cashA $:=0$. To prevent empty_cash from interfering with the invariant above, we could add a guard to empty_cash specifying that it must not happen while there is a transaction in progress in the ATM.

\section{Related Work}

In this section we outline some of the work related to refinement of UML diagrams. The work on state machines refinement has been introduced by Snook and Walden in [12]. Their work is based on the old version of UMLB [11] which was based on classical B and has been extended to include translation to an event "style" of B (which was a precursor to Event-B). They introduced state elaboration and transition elaboration techniques. The semantics of the state machine refinement are given by Event-B. However, we provide a more precise definition of refined state machine and we provide tool support based on UML-B giving a different model visualisation from the UML diagram symbol used in [12]. We also introduce class refinement techniques which are not dealt with in [12]. In [13], Plaska et al. have suggested a process for refinement involving the application of patterns that are based on the techniques introduced in $[12]$.

The techniques of adding new attributes and associations to a class and adding new classes to a class diagram have been introduced in informal way for refinement of UML class diagram [15] but no formal notation nor formal refinement concept is used. Templates are introduced for attributes and associations to specify the translation of model elements to low level design and implementation. [15] has also discussed on possible tool support for the templates. Also, the technique of state elaboration has been introduced in a refinement of UML state diagram [14] again without a formal notion of refinement. Simons [19] has presented four informal refinement rules of state machines. The rules in the refinements are: (1) New states must be sub-states nested in the abstract states (super-states), (2) New transitions must only connect between the sub-states, (3) The incoming and outgoing transitions of the superstates must be preserved, and (4) The self transitions of the super-states must be preserved. Rules (1) and (2) must also be followed in UML-B state machine refinement. These two rules are achieved by applying the state elaboration technique. Rule (3) must also be followed in UML-B for a state machine refinement to be valid. In contrast to Rule (4), in our work, when refining self transitions, the occurrence of the transitions can either be many times or can be restricted to once. Restriction to once means removing looping behaviour and this is a valid refinement since we focus on preserving safety, not liveness, in our work. Unlike our work, Simon's work does not involve any formal notion and does not discuss any tool supporting the rules.

There is much more work on combining UML with formal notations and we now outline some of this. However, unlike our work, none of this work supports refinement in UML to the best of our knowledge. Lano, Clark and Androutsopoulos [16] present the translation of UML-RSDS into classical B. The work focused on translating class diagrams into B. Each class is translated into a respective B machine. Unlike UML-B, where all classes in a class diagram are translated into one Event$\mathrm{B}$ machine. The constraint language used is OCL whereas we use $\mu \mathrm{B}$. Idani, Ledru and Bert [17] have investigated the reverse in which they proposed an approach and tool support for the construction of UML diagrams from B specifications. Ledang and Souquiéres have introduced an approach for translating UML state machine diagrams into classical B in [20]. The translations use the state function representation whereas UML-B supports both state function and state sets representations. Mammar and Laleau [22] have also work on the translation of class and state diagrams into Classical B. Their work is suitable for a development of data-oriented applications in contrast to our work which is suitable for process-oriented applications. Another difference is that the refinements involve the generated abstract $\mathrm{B}$ models and there is no concept of refinement in UML whereas, in our work the refinements involve the class and state machine diagrams. Laleau and Polack [36] have extended the meta-model of UML class diagrams specifically for information system specifica- 
tion. The semantics of the extended meta-model are defined in B invariants. The invariants formalise the associations in the meta-model and rules for integrity constraints. In [37], tools to translate from UML class diagrams into B machines and vice versa have been developed applying the extended meta-model and semantics in [36]. However, [36] and [37] do not deal with refinement. Knapp et al. [31] have investigated the validity of UML state machine refinements by formalizing with MTLA [32]. In contrast to our work, their work does not consider state machine hierarchy in refinements. New transitions and states may be introduced in a refined state machine by replacing old states with new states and transitions. We prefer our approach because the relationship between abstract state machines and refinements is clearer. In UML-B, new transitions and states may be added in nested state machines. UML-B is more restrictive but this makes the refinement pattern simple and clear. Similar to our work, refining self transitions may be restricted to once as the work does not focus on liveness properties.

Integration work of UML with $\mathrm{Z}$ has also been investigated. Moller et al. [26] have integrated the formal method, namely CSP-OZ [27] into UML and Java. A UML profile for CSP-OZ is developed. A UML profile contains an extension mechanism that consists of stereotype and tag definitions. This profile which integrates UML and CSP-OZ is similar to the UML$B$ profile [28] of previous version of UML-B. In this work, class diagrams, state machines and the UML-RT structure diagrams are translated to CSP-OZ (an integrated formal method) specifications. Amalio et al. [18] also have investigated an integration between UML and Z. They have introduced a framework called UML $+\mathrm{Z}$ for building, analysing and refining models bases on UML and Z. UML $+\mathrm{Z}$ models consists of class, state and object diagrams. The integration work of UML and VDM has been done by Frey [23]. Frey has introduced a methodology where UML and VDM-SL are used together in modelling to take advantage of both notations. Lausdahl et al. [21] have work on a bi-directional translation between UML class diagram and VDM++. The translation of the sequence diagram is done from UML to $\mathrm{VDM}++$. The translations are implemented as a plug-in to the Overture [24] toolsets.

In section 7 we discussed two simple approaches to finding gluing invariants, both of which rely on UMLB state machine refinement. Llano et al. [38] propose a method to discover Event-B gluing invariants using automated theory formation rather than failed proof obligation. Their approach is more complicated than ours, requiring the construction of data tables from simulation traces of the model, but is more general since it does not rely on the style of refinement imposed by UML-B. In [39], Ireland et al. examine failed proof obligations but focus on finding omissions from the model and do not focus on refinement. In contrast, our approach assumes the model is correct and only attempts to find a gluing invariant.

\section{Conclusions}

In [25] we have introduced notions of refined class, refined state machine and extended classtype for UML-B. We used these notions to describe the following refinement techniques:

- Add new attributes and associations to a refined class

- Add new classes in a refinement

- State elaboration

- Transition elaboration

- Add new attributes and associations to an extended classtype.

- Add new classtypes in a refinement.

In this paper, we provide a more extensive account of UML-B refinement techniques. We give a formalisation for UML-B refinement rules and describe the extensions to the UML-B meta-model which gives precise definition of the notions of refined class, refined state machine and extended classtype. The meta-model is used to extend the UML-B drawing tools. We have applied the UML-B meta-model extensions in the ATM case study. The Rodin tool was used to generate and prove the proof obligations. Based on the ATM case study, we provide two ways of constructing gluing invariants.

One area that currently lacks support in UML-B is parallel state-machines. Although UML-B has support for modelling parallel state machines within one refinement level, the transition elaboration mechanism does not allow the parallel state machines to be linked correctly with their parent state-machines. In future, we will extend the UML-B meta-model to support refinement of parallel state machines.

Our experience using UML-B for modelling refinements has been that the proof of refinements is comparatively straightforward compared with working directly with Event-B. This may be due to the organisational structures imposed by the patterns generated by UMLB. For example the explicit annotation of states that was discussed in the section on designing gluing invariants. This is an interesting area for future work including extending the approaches to constructing gluing invariants to other refinement patterns of UML-B and comparing ease of proof with equivalent models written directly and freely in Event-B. Further case studies 
are needed to explore this as well as to further validate the existing techniques and develop new extensions to UML-B refinement.

We are currently working on supporting decomposition concepts in UML-B. Decomposition is needed to ensure scalability of the method. Event-B machines may be decomposed into several sub-machines in such a way that each sub-machine can be refined individually while preserving the overall validity of refinement. That is, if the sub-machines were re-composed the composition would be a valid refinement of the original machine before it was decomposed. We have previously [40] introduced techniques to refine UML-B state machines in a way that prepares for decomposition and have introduced the concept of a UML-B composed machine to define the composition of a number of UML-B submachines. Our current work is to develop the UML-B composed machine, extending its features to fully support Event-B decomposition techniques in UML-B. We are also extending the UML-B tooling to visualise the UML-B composed machine.

Acknowledgements We would like to thank the reviewers for their insightful comments and feedback on this paper.

\section{References}

1. Snook, C. and Butler, M. : UML-B and Event-B: An Integration of Languages and Tools. In: The IASTED International Conference on Software Engineering, pp. 336-341.(2008)

2. Object Management Group: Introduction to OMG's Unified Modelling Language (UML). http://www.omg.org/gettingstarted/what_is_uml.htm. Date Last Accessed:23/8/13.

3. Rumbaugh, J., Booch, G. and Jacobson, I.: The Unified Modelling Language User Guide, Addison Wesley. (1999)

4. Metayer, C., Abrial, J.R., Voisin, L.: Event-B Language. Technical Report Deliverable 3.2, EU Project IST-511599 - RODIN, http://rodin.cs.ncl.ac.uk/deliverables/D7.pdf. Date Last Accessed: 25/1/08.(2005)

5. Abrial, J. R., Butler, M., Hallerstede, S., Hoang, T. S., Mehta, F. and Voisin, L.: Rodin: An Open Toolset for Modelling and Reasoning in Event-B, International Journal on Software Tools for Technology Transfer. vol. 12, pp. 447-466, Springer.(2010)

6. Abrial, J. : The B-Book: Assigning Programs to Meanings, Cambridge University Press. (1996)

7. Abrial, J. : Modeling in Event-B - System and Software Engineering, Cambridge University Press. (2010)

8. Abrial, R. and Hallerstede, S.: Refinement, Decomposition and Instantiation of Discrete Models: Application to Event-B, Journal Fundamentae Informatica. vol. 77, issue 1-2, pp. 1-28, IOS Press. (2007)

9. Butler, M. and Yadav, D.: An Incremental Development of the Mondex System in Event-B, Journal Formal Aspects of Computing, vol. 20, issue 1, pp. 61-77, Springer. (2008)

10. Butler, M. and Hallerstede, S.: The Rodin Formal Modelling Tool, BCS-FACS Christmas 2007 Meeting, Formal Methods In Industry, London.(2007)
11. Snook, C. and Butler, M., UML-B: Formal Modelling and Design Aided by UML, ACM Transactions on Software Engineering and Methodology, vol. 15, issue 1, pp. 92-122, ACM Press. (2006)

12. Snook, C. and Walden, M. : Refinement of Statemachines Using Event B Semantics, B2007: Formal Semantic and Development in B, LNCS 4355, pp. 171-185, Springer. (2006)

13. Plaska, M., Walden, M. and C. Snook: Documenting the Progress of the System Development. In: Proc. of Workshop on Methods, Models and Tools for Fault Tolerance, pp. 251 274 , Springer. (2007)

14. Object Management Group: UML 2.1.2 Superstructure Specification. http://www.omg.org/cgi-bin/docs/formal/200711-02.pdf. Date Last Accessed:23/8/13.

15. Bergner, K., Rausch, A., Sihling, M. and Vilbig, A.: Structuring and Refinement of Class Diagrams. In: The 32nd Annual Hawaii International Conference, vol. 6, pp. 6018. (1999)

16. Lano, K., Clark, D. and Androutsopoulos, K.: UML to B: Formal Verification of Object Oriented Models. In: International Conference of Integrated Formal Method, pp. 761-768, Springer. (2004)

17. Idani, A. Ledru, L. and Bert, D.: Derivation of UML Class Diagrams as Static Views of Formal B Developments, In: International Conference on Formal Engineering Methods, pp. 37-51, Springer. (2005)

18. Amálio, N., Polack, F. and Stepney, S. : UML + Z: Augmenting UML with Z, In: Software Specification Methods, pp.81 102, Hermes Science Publishing. (2006)

19. Simons, A. J. H.: A Theory of Regression Testing for Behaviourally Compatible Object Types, Journal Software Testing, Verification and Reliability, vol. 16, issue. 3, pp. 133-156, John Wiley and Sons Ltd. (2006)

20. Ledang, H. and Souquiéres, J. : Contributions for Modelling UML State-Charts in B. In: International Conference of Integrated Formal Methods, LNCS 2335, pp. 109-127, Springer. (2002)

21. Lausdahl, K. G., Lintrup, H. K. A. and Larsen, P. G.: Coupling Overture to MDA and UML. Master Thesis. (2008)

22. Mammar, A. and Laleau, R.: A Formal Approach Based on UML and B for the Specification and Development of Database Application, Journal Automated Software Engineering, vol. 13, issue 4, pp 497-528, Springer. (2006)

23. Frey, P.: Combining UML Use Cases and VDM-SL,Paper for the Seminar in Software Technology at the Institute for Software Technology (IST), Graz University of Technology, Austria. (2000)

24. Larsen P. G., Battle N., Ferreira M., Fitzgerald J., Lausdahl, K. and Verhoef, M.: The Overture Initiative Integrating Tools for VDM, Journal SIGSOFT Softw. Eng. Notes, vol. 35, issue 1, pp. 1-6, ACM. (2010)

25. Said, M. Y., Butler, M. and Snook, C.: Language and Tool Support for Class and State Machine Refinement in UML-B, In: Internation Conference of Formal Methods, LNCS 5850, pp.579-595, Springer. (2009)

26. M. Moller, E. Olderog, H. Rasch and H. Wehrheim,: Linking CSP-OZ with UML and Java: A Case Study. In: International Conference of Integrated Formal Methods, LNCS2999, pp. 267286, Springer. (2004)

27. Fischer, C.: CSP-OZ: A Combination of Object-Z and CSP, Technical Report. University of Oldenburg, Germany. (1997)

28. Snook, C., Butler, M. and Oliver, I.: The UML-B Profile for Formal Systems Modelling in UML, In: UML-B Specification for Proven Embedded Systems Design, pp 69-84, Springer. (2004)

29. The Eclipse Foundation: "Eclipse Modelling Framework", http://www.eclipse.org/emf/. Date Last Accessed: 07/08/2013 
30. The Eclipse Foundation: "Graphical Modelling Project", http://www.eclipse.org/gmp/. Date Last Accessed: 07/08/2013

31. Knapp, A., Merz, S. and Wirsing, M.: "Refining Mobile UML State Machines", LNCS3116, pp 274-288, Springer. (2004)

32. Merz, S., Wirsing, M. and Zappe, J.: A Spatio-Temporal Logic for the Specification and Refinement of Mobile Systems, LNCS 2621, pp 87-101, Springer. (2003)

33. Snook, C., Savicks, V. and Butler, M.: Verification of UML models by translation to UML-B. In International Conference of Formal methods for Components and Objects, LNCS6957, pp 251-266, Springer. (2012)

34. Snook, C., Fritz, F. and Illisaov, A. An EMF Framework for Event-B. In: Workshop on Tool Building in Formal Methods ABZ Conference, Orford, Quebec, Canada. (2010)

35. The Object Management Group: "Meta Object Facility (MOF) 2.0 Query/View/Transformation (QVT)", http://www.omg.org/spec/QVT/. Date Last Accessed: 07/08/2013

36. Laleau, R. and Polack, F.: A Rigorous Metamodel for UML Static Conceptual Modelling of Information Systems. In: International Conference on Advanced Information Systems Engineering, LNCS 2068, pp. 402-416, Springer. (2001)

37. Laleau, R. and Polack, P.: Coming and Going from UML to B: A Proposal to Support Traceability in Rigorous IS Development. In: International Conference of B and Z, LNCS2272, pp 517-534, Springer. (2002)

38. Llano, M. T., Ireland, A. and Pease, A.: Discovery of Invariants through Automated Theory Formation, Formal Aspects of Computing, pp. 1-47, Springer. (2012)

39. Ireland, A., Grov, G. and Butler, M.: Reasoned Modelling Critics: Turning Failed Proofs into Modelling Guidance, In : International Conference of Abstract State Machines, Alloy, B and Z, LNCS 5977, 189-202, Springer. (2010)

40. Said, M.Y.: Methodology of Refinement and Decomposition in UML-B, PhD Thesis, University of Southampton. (2010) 